TITLE:

\title{
Formulation and implementation of direct algorithm for the symmetry-adapted cluster and symmetry-adapted cluster- configuration interaction method
}

\section{AUTHOR(S):}

Fukuda, Ryoichi; Nakatsuji, Hiroshi

\section{CITATION:}

Fukuda, Ryoichi ...[et al]. Formulation and implementation of direct algorithm for the symmetry-adapted cluster and symmetry-adapted cluster-configuration interaction method. JOURNAL OF CHEMICAL PHYSICS 2008, 128(9): 094105.

\section{ISSUE DATE:}

2008-03-07

URL:

http://hdl.handle.net/2433/84613

\section{RIGHT:}

Copyright 2008 American Institute of Physics. This article may be downloaded for personal use only. Any other use requires prior permission of the author and the American Institute of Physics. 


\title{
Formulation and implementation of direct algorithm for the symmetry- adapted cluster and symmetry-adapted cluster-configuration interaction method
}

\author{
Ryoichi Fukuda ${ }^{1,2, a)}$ and Hiroshi Nakatsuji ${ }^{1,2, b)}$ \\ ${ }^{1}$ Quantum Chemistry Research Institute, ${ }^{\mathrm{c}}{ }^{2}$ Kyodai Katsura Venture Plaza, North Building 106, \\ 36-1 Goryo Oohara, Nishikyo-ku, Kyoto 615-8245, Japan \\ ${ }^{2}$ Department of Synthetic Chemistry and Biological Chemistry, Graduate School of Engineering, \\ Kyoto University, Kyoto-Daigaku-Kaysura, Nishikyo-ku, Kyoto 615-8510, Japan
}

(Received 26 July 2007; accepted 14 December 2007; published online 4 March 2008)

\begin{abstract}
We present a new computational algorithm, called direct algorithm, for the symmetry-adapted cluster (SAC) and SAC-configuration interaction (SAC-CI) methodology for the ground, excited, ionized, and electron-attached states. The perturbation-selection technique and the molecular orbital index based direct sigma-vector algorithm were combined efficiently with the use of the sparse nature of the matrices involved. The formal computational cost was reduced to $O\left(N^{2} \times M\right)$ for a system with $N$-active orbitals and $M$-selected excitation operators. The new direct SAC-CI program has been applied to several small molecules and free-base porphin and has been shown to be more efficient than the conventional nondirect SAC-CI program for almost all cases. Particularly, the acceleration was significant for large dimensional computations. The direct SAC-CI algorithm has achieved an improvement in both accuracy and efficiency. It would open a new possibility in the SAC/SAC-CI methodology for studying various kinds of ground, excited, and ionized states of molecules. (C) 2008 American Institute of Physics. [DOI: 10.1063/1.2832867]
\end{abstract}

\section{INTRODUCTION}

The symmetry-adapted cluster $(\mathrm{SAC})^{1}$ and $\mathrm{SAC}-$ configuration interaction $(\mathrm{SAC}-\mathrm{CI})^{2}$ methodology proposed and first coded by one of the authors in 1978 is an electron correlation methodology for ground, excited, ionized, and electron-attached states of molecules. It has been successfully applied to diverse chemistry, physics, and biology involving various kinds of electronic and vibrational states (for recent reviews, see Ref. 3). The methodology is based on the cluster expansion formalism combined with the variational principle. ${ }^{2}$ Later, theoretically identical methodologies, such as coupled-cluster linear response theory ${ }^{4}$ and equation-ofmotion coupled cluster, ${ }^{5}$ have been reported. These methods established a highly accurate way to study excited electronic structures of molecules.

The earlier version of the SAC/SAC-CI program ${ }^{6}$ was published in 1985. A great deal of effort has been made to the theory and program development, and a lot of new ideas have been implemented. The SAC-CI general- $R$ method, ${ }^{7}$ multireference version of the SAC/SAC-CI method, ${ }^{8}$ exponentially generated wave function idea, ${ }^{9}$ extension up to septet spin multiplicity, ${ }^{10}$ and the analytical energy gradient method $^{11}$ were the representative fruits of these efforts. Recently, the giant SAC/SAC-CI theory for giant molecular crystals has been proposed. ${ }^{12}$ The SAC/SAC-CI method has been implemented in the GAUSSIAN03 software program package

\footnotetext{
${ }^{\text {a) }}$ Electronic mail: fukuda@qcri.or.jp.

${ }^{b)}$ Electronic mail: h.nakatsuji@qcri.or.jp.

${ }^{c)}$ URL: http://www.qcri.or.jp/.
}

and has been widely used in universities and industries. ${ }^{13}$ So far, the SAC/SAC-CI code has been written with the excitation-operator driven algorithm ${ }^{1,2}$ with the use of the perturbation-selection technique. ${ }^{14,15}$ The integral driven algorithm was introduced to the SAC/SAC-CI method by Hirao, ${ }^{16}$ but his algorithm was not combined with the perturbation-selection technique.

Molecular orbital (MO) integral driven direct algorithm for electron correlation methods was first proposed by Roos for singles and doubles (SD) CI method. ${ }^{17}$ In such a procedure, the iteration vector (usually termed as sigma vector) is directly constructed from MO integrals, without an explicit construction of a Hamiltonian matrix. At the same time, when the SAC/SAC-CI SD code was established, ${ }^{1,2}$ the coupled-cluster doubles (CCD) method only for ground state was formulated by Pople et al. in integral driven form. ${ }^{18} \mathrm{An}$ efficient computation algorithm of CCSD was designed by Scuseria et al. ${ }^{19}$ who introduced intermediate arrays for efficiency. These MO integral driven approaches include all $\mathrm{SD}$ or D excitation operators. We consider that this feature conflicts with the policy of our SAC/SAC-CI program explained below.

A policy of our SAC/SAC-CI program is that we discard minor unimportant terms by introducing some selection procedures with thresholds. Accordingly, depending on the desired accuracy, we introduce appropriate thresholds of selections, and less important but time-consuming terms are neglected. ${ }^{20}$ The perturbation selection of the linked operators is a particularly important technique. ${ }^{14,15}$ By virtue of this technique, we can use a single theory and a single program for various research subjects; ${ }^{3}$ from fine theoretical 
spectroscopy of small molecules ${ }^{21-23}$ to photobiology of big biological molecules. ${ }^{24,25}$ This feature enables us to investigate various chemical phenomena from an equal viewpoint. The perturbation-selection technique is particularly important for excited states. The atomic orbital (AO) basis functions are usually optimized for the ground state. We need a flexible AO basis to describe various kinds of excited states. Diffuse functions and/or sizable Rydberg functions are often required. Therefore, the number of active $\operatorname{MOs}(N)$ for excited-state calculations is much larger than that for ground state. Order $N^{6}$ algorithms (the optimal scale of CCSD method) will soon face the limit of computer. The limitations of $O\left(N^{6}\right)$ algorithms would be much more severe for excited states than for ground states.

A problem in the algorithm of the conventional SAC-CI program lies in the calculations of unlinked terms. The Hamiltonian matrix elements between selected excitation operators are evaluated within the loops driven by the labels of excitation operators. Consequently, the unlinked integral part becomes an $O\left(M^{3}\right)$ step, where $M$ is the number of selected excitation operators. This step is so time consuming that the additional approximation shall be introduced for practical calculations, and the approximation was to cut less important excitation operators than a given threshold off the loops. This cutoff approximation is, however, problematic because the unlinked terms are essentially important in the SAC/SAC-CI theory.

An efficient use of the core memory is also very important. We use a projective reduction formula ${ }^{26,27}$ to evaluate the Hamiltonian matrix elements between selected excitation operators. The MO integrals are randomly requested from the projective reduction routine in the loops of the excitation operator labels. Therefore, we have to load all the MO electron repulsion integrals (ERIs) on a core memory. If the core memory is not enough to load them on, we have to use a multipass algorithm for separated MO ERIs, but this multipass algorithm is inefficient.

In this paper, a solution for these problems is provided by combining the direct algorithm with the perturbationselection technique. We have developed the direct SAC and SAC-CI algorithm running within the selected excitation operators. The MO based direct algorithm that utilizes the sparseness of the Hamiltonian matrices provides an efficient algorithm that works within the selected excitation operators. This also results in an efficient minimal memory requirement.

The theoretical framework and the algorithm of the direct SAC/SAC-CI method are described in the next section. The implementation of the new direct SAC-CI algorithm is given in Sec. III, and applications to several test cases are reported in Sec. IV. The present article focuses on singlepoint calculations. The analytical energy gradient of the SAC/SAC-CI method has been given in GAUSSIAN 03. We have also adapted the direct algorithm for the analytical energy gradient calculations, and it will be reported in the subsequent paper.

\section{THEORY}

\section{A. SAC/SAC-CI method}

The details of the SAC/SAC-CI methodology have been reviewed in several articles. ${ }^{1-3}$ Here, we summarize the points pertinent to the present study. The SAC expansion for a totally symmetric singlet ground state is written as

$$
\Psi_{\text {SAC }}=\exp (S)|0\rangle
$$

where $|0\rangle$ is a closed-shell Hartree-Fock (HF) single determinant and

$$
S=\sum_{I} c_{I} S_{I}^{\dagger}
$$

$S_{I}^{\dagger}$ is a symmetry-adapted excitation operator. The SAC coefficient $c_{I}$ is calculated by solving the nonvariational equations

$$
\left\langle 0\left|\left(H-E_{\text {SAC }}\right)\right| \Psi_{\text {SAC }}\right\rangle=0
$$

and

$$
\left\langle 0\left|S_{L}\left(H-E_{\text {SAC }}\right)\right| \Psi_{\text {SAC }}\right\rangle=0,
$$

where $H$ is the Hamiltonian and $E_{\mathrm{SAC}}$ is the SAC energy.

The SAC theory provides not only the SAC wave function $\Psi_{\text {SAC }}$, but also a set of functions that constitute the basis for the excited states. Namely, the set of functions

$$
\Phi_{K}=P S_{K}^{\dagger}\left|\Psi_{\text {SAC }}\right\rangle
$$

provides an adequate basis for expanding the excited states. Here, $P$ is a projection operator $P=1-\left|\Psi_{\text {SAC }}\right\rangle\left\langle\Psi_{\text {SAC }}\right|$. Therefore, we expand our excited states by linear combinations of $\left\{\Phi_{K}\right\}$ as

$$
\Psi_{\mathrm{SAC}-\mathrm{CI}}^{(p)}=\sum_{K} d_{K}^{(p)} \Phi_{K}
$$

which is the SAC-CI wave function for the excited states. Here, the index $p$ labels the excited state. The SAC-CI wave function can also be defined for the excited state having different symmetries and for the ionized and electron-attached states. Including these states, Eq. (5) is generalized as

$$
\Phi_{K}=P R_{K}^{\dagger}\left|\Psi_{\text {SAC }}\right\rangle,
$$

where $\left\{R_{K}^{\dagger}\right\}$ denotes a set of excitation, ionization, and electron-attachment operators. The SAC-CI coefficients and energies are calculated by solving the nonvariational equation

$$
\left\langle 0\left|R_{L}\left(H-E_{\text {SAC-CI }}^{(p)}\right)\right| \Psi_{\text {SAC-CI }}^{(p)}\right\rangle=0 .
$$

In the SAC/SAC-CI program, we can use the perturbation-selection method. We select only important excitation operators by the second-order perturbation theory and reduce the labors in the SAC and SAC-CI calculations. ${ }^{14,15,20}$ In the singles and doubles method (SAC/ SAC-CI SD-R), we include all singles and apply a perturbation selection to doubles. In the ground state SAC calculation, the doubly excitation operator $S_{K}^{\dagger}$ is included if 


$$
\left|E_{s}\left(S_{K}^{\dagger}\right)\right| \geqslant \lambda_{g},
$$

where $E_{s}\left(S_{K}^{\dagger}\right)$ is the second-order energy contribution of $S_{K}^{\dagger}$ to the ground state,

$$
E_{s}\left(S_{K}^{\dagger}\right)=\frac{\left\langle 0\left|S_{K} H\right| 0\right\rangle\left\langle 0\left|H S_{K}^{\dagger}\right| 0\right\rangle}{\left\langle 0\left|S_{K} H S_{K}^{\dagger}\right| 0\right\rangle-\langle 0|H| 0\rangle} .
$$

For excited, ionized, and electron-attached states, the doubly excitation operators which satisfy

$$
\left|E_{p}\left(R_{K}^{\dagger}\right)\right| \geqslant \lambda_{e}
$$

with

$$
E_{p}\left(R_{K}^{\dagger}\right)=\frac{\left\langle 0\left|R_{K} H\right| \Psi_{\mathrm{Ref}}^{(p)}\right\rangle\left\langle\Psi_{\mathrm{Ref}}^{(p)}\left|H R_{K}^{\dagger}\right| 0\right\rangle}{E_{\text {Ref }}^{(p)}-\left\langle 0\left|R_{K} H R_{K}^{\dagger}\right| 0\right\rangle}
$$

are included in the SAC-CI calculations. Here, $\Psi_{\text {Ref }}^{(p)}$ is the reference wave function which has the energy $E_{\mathrm{Ref}}^{(p)}$. The recommended usage of the perturbation-selection technique is found in the SAC-CI Guide. ${ }^{20}$

\section{B. Conventional nondirect SAC/SAC-CI algorithm}

The conventional version of the SAC/SAC-CI program was formulated to be driven with the excitation operator labels. For example, the SAC-CI secular equation is written as

$$
\sum_{K}\left(H_{L K}+U_{L K}\right) d_{K}^{(p)}=E_{\mathrm{SAC}-\mathrm{Cl}}^{(p)} \sum_{K} d_{K}^{(p)} S_{L K} .
$$

Here, $H_{L K}, U_{L K}$, and $S_{L K}$ denote linked, unlinked, and overlap matrix elements, respectively,

$$
\begin{aligned}
& H_{L K}=\left\langle 0\left|R_{L} H R_{K}^{\dagger}\right| 0\right\rangle, \\
& U_{L K}=\sum_{I}\left\langle 0\left|R_{L} H R_{K}^{\dagger} S_{I}^{\dagger}\right| 0\right\rangle c_{I},
\end{aligned}
$$

and

$$
S_{L K}=\left\langle 0\left|R_{L} R_{K}^{\dagger}\right| 0\right\rangle .
$$

For simplicity, we dropped higher-order unlinked terms in Eqs. (13) and (15) and the projection operator that will appear in Eq. (16). The details of this simplification were described in Ref. 20. The generalized eigenvalue problem of Eq. (13) is solved iteratively with the modified Davidson's procedure, ${ }^{28}$ where the sigma vector,

$$
\sigma_{L}^{(m)}=\sum_{K}\left(H_{L K}+U_{L K}\right) b_{K}^{(m)},
$$

is constructed in each iteration step from the basis vector $\mathbf{b}$ and the matrix elements. In the conventional code, the matrix elements are evaluated with the projective reduction formula and are stored on disk. The loops for the integral evaluation [Eqs. (14)-(16)] and the sigma-vector construction [Eq. (17)] are driven through the excitation operator labels $I, L$, and $K$. We call this algorithm conventional "nondirect" algorithm, in contrast to the "direct" algorithm introduced in this paper.

The important features of the conventional nondirect algorithm are as follows:

(1) The perturbation-selection technique is easily introduced into the program. The perturbation selection re- duces the number of the matrix elements to be evaluated and the range of the summation in Eqs. (13) and (17), thus dramatically reducing the total computation time.

(2) The extensions to include general excitation operators are straightforward. This feature was important for the generalizations of the SAC-CI code, like the SAC-CI general- $R$ method ${ }^{7}$ and SAC-CI for high-spin multiplicities. ${ }^{10}$ These extensions were done by expanding the nature of the excitation operators and could be easily implemented in the conventional code by expanding the loops of the operators labels $I, L$, and $K$. The matrix elements are calculated using the projective reduction formula, PROJR. ${ }^{27}$ The PROJR program evaluates the matrix elements by comparing the left and right configuration state functions. The algorithm represented by Eqs. (14)-(17) does not depend on the specific form of the $R$ operators.

Because of these attractive features, the conventional SAC-CI program has been extended to cover a wide range of chemistry and accuracy ${ }^{3}$ and has been successfully applied from fine spectroscopy ${ }^{21-23}$ of rather small molecules to biospectroscopy ${ }^{24}$ and photobiology involving moderately large molecules. ${ }^{25}$ However, the conventional algorithm has following demerits in comparison with the direct algorithm introduced in this paper.

(1) The calculation is time consuming, particularly for the unlinked terms. The unlinked terms $U$ in Eq. (15) have three indices of excitation operators, which result in a triply nested loop structure. However, many of the terms, $\left\langle R_{L}|H| R_{K}^{\dagger} S_{I}^{\dagger}\right\rangle$, are identically zero because of Slater's rule, but the calculation of these unlinked terms can be done only at the innermost part of the loop.

(2) A whole MO ERI has to be loaded on core memory. This is because the MO ERI is randomly accessed from the PROJR subroutine. There is no regulation in the access of the MO ERI.

In constructing the direct SAC/SAC-CI code, we want to overcome these demerits, keeping the merits of the perturbation selection.

\section{Direct SAC/SAC-CI algorithm}

In the direct SAC method, the excitation operators $S^{\dagger}$ and the coefficients $c$ are defined by the MO labels instead of the excitation operator labels. For singles $\left(S_{1}\right)$ and doubles $\left(S_{2}\right)$, they are

$$
S^{\dagger}=S_{1}+S_{2}=\sum_{i} \sum_{a} c_{i}^{a} S_{i}^{a \dagger}+\frac{1}{2} \sum_{i j} \sum_{a b} c_{i j}^{a b} S_{i j}^{a b \dagger} .
$$

Hereafter, we use indices $i, j, k, \ldots$ for occupied MOs, $a, b, c, \ldots$ for virtual MOs, and $p, q, r, s, \ldots$ for general MO's. Inserting Eq. (18) into Eqs. (3) and (4), we obtain the expressions of the SAC energy, the SAC equations for $S_{1}$ and $S_{2}$. In the direct SAC algorithm, we first write down these working equations explicitly as summarized in Table $\mathrm{X}$ of the Appendix, where $f_{q}^{p}=\langle p|f| q\rangle$ and $v_{q s}^{p r}=(p q \mid r s)=\langle p r \mid q s\rangle$ 
denote the Fock matrix element and the MO ERI, respectively. We introduced the permutation operator

$$
\mathcal{P}_{i j}^{a b}[\cdots]_{i j}^{a b}=[\cdots]_{i j}^{a b}+[\cdots]_{j i}^{b a}
$$

for convenience. Note that the expressions in Table $\mathrm{X}$ are not unique. We will introduce intermediate arrays to make the number of operations minimal. ${ }^{19}$ The expressions in Table $\mathrm{X}$ were designed to be effective in the perturbation-selection technique.

The SAC-CI secular equation [Eq. (8)] is written in the nonsymmetric eigenvalue problems

$$
\mathbf{H d}^{(p)}=E_{\mathrm{SAC}-\mathrm{CI}}^{(p)} \mathbf{S \mathbf { d } ^ { ( p ) }}
$$

and

$$
\mathbf{H}^{*} \overline{\mathbf{d}}^{(p)}=E_{\mathrm{SAC}-\mathrm{CI}}^{(p)} \mathbf{S}^{*} \overline{\mathbf{d}}^{(p)},
$$

where $\mathbf{d}$ and $\overline{\mathbf{d}}$ denote the right-hand and left-hand eigenvectors, respectively. The superscript $*$ denotes a Hermitian transpose. In the generalized Davidson procedure, ${ }^{28}$ we use $m$ basis vectors $\mathbf{b}_{i}$ and $\overline{\mathbf{b}}_{i}$, which are collected in matrices $\mathbf{B}$ and $\overline{\mathbf{B}}$ as

$$
\mathbf{B}=\left(\mathbf{b}_{1}, \mathbf{b}_{2}, \ldots, \mathbf{b}_{m}\right), \quad \overline{\mathbf{B}}=\left(\overline{\mathbf{b}}_{1}, \overline{\mathbf{b}}_{2,} \ldots, \overline{\mathbf{b}}_{m}\right) .
$$

Here, $m$ is some small number used in generalized Davidson's procedure. ${ }^{28}$ The basis vectors $\mathbf{b}_{i}$ and $\overline{\mathbf{b}}_{i}$ satisfy the biorthonormal relation

$$
\overline{\mathbf{B}} * \mathbf{S B}=\mathbf{1},
$$

where $\mathbf{1}$ is an $m \times m$ unit matrix. Then, we form a small Davidson matrix as

$$
\widetilde{\mathbf{H}}=\overline{\mathbf{B}}^{*} \mathbf{H B}=\overline{\mathbf{B}}^{*} \mathbf{\Sigma}=\overline{\mathbf{\Sigma}}^{*} \mathbf{B} .
$$

The elements of $\boldsymbol{\Sigma}=\mathbf{H B}$ and $\overline{\boldsymbol{\Sigma}}=\mathbf{H}^{*} \overline{\mathbf{B}}$ are the so-called sigma vectors for the right-hand and left-hand basis vectors, respectively. Explicitly, they are

$$
\boldsymbol{\sigma}_{i}=\mathbf{H b} \mathbf{b}_{i}, \quad \overline{\boldsymbol{\sigma}}_{i}=\mathbf{H}^{*} \overline{\mathbf{b}}_{i} .
$$

To impose a biorthonormal relation to the basis, we define tau vectors as

$$
\boldsymbol{\tau}_{i}=\mathbf{S} \mathbf{b}_{i}, \quad \overline{\boldsymbol{\tau}}_{i}=\mathbf{S} * \overline{\mathbf{b}}_{i} .
$$

In each iteration, the small matrix

$$
\overline{\mathbf{C}}^{*} \widetilde{\mathbf{H}} \mathbf{C}=\mathbf{D}
$$

is diagonalized. After convergence, we obtain the SAC-CI vectors by the following expression:

$$
\mathbf{d}=\mathbf{B C}, \quad \overline{\mathbf{d}}=\overline{\mathbf{B}} \overline{\mathbf{C}} .
$$

In the SAC-CI SD- $R$ method, the sigma and tau vectors have three blocks: $R_{0}(\mathrm{HF}), R_{1}$ (singles), and $R_{2}$ (doubles) as

$$
\boldsymbol{\sigma}=\left(\begin{array}{c}
\sigma_{0} \\
\sigma_{1} \\
\sigma_{2}
\end{array}\right)=\left(\begin{array}{c}
\sigma_{0} \\
\sigma_{i}^{a} \\
\sigma_{i j}^{a b}
\end{array}\right) .
$$

The MO-indexed representations of the working equation are obtained from Eq. (8) by using the MO-indexed $R$ operators. For singlet excited states, the $R$ operators are

$$
R^{\dagger}=R_{0}+R_{1}+R_{2}=d_{0}+\sum_{i} \sum_{a} d_{i}^{a} R_{i}^{a \dagger}+\frac{1}{2} \sum_{i j} \sum_{a b} d_{i j}^{a b} R_{i j}^{a b \dagger} .
$$

The SAC-CI coefficients are obtained from the basis $\mathbf{b}$ as follows:

$$
d_{i j}^{a b}=\sum_{m} b_{i j}^{a b(m)} C^{(m)} .
$$

The resultant working equations for the singlet excited states are summarized in Table XI of the Appendix. For the lefthand projection, we use the following notation for the Hermite conjugation:

$$
\left(\bar{\sigma}_{i j}^{a b}\right)^{*}=\bar{\sigma}_{a b}^{i j}, \quad\left(\bar{b}_{i j}^{a b}\right)^{*}=\bar{b}_{a b}^{i j} .
$$

To simplify our representation, upper bars were dropped for the left-hand projections in Table XI.

For triplet states, the MO-indexed $R$ operator is

$$
R^{\dagger}=R_{1}+R_{2}=\sum_{i} \sum_{a} d_{i}^{a} R_{i}^{a \dagger}+\sum_{i j} \sum_{a \neq b} d_{i j}^{a b} R_{i j}^{a b \dagger} .
$$

The working equations for the triplet states are summarized in Table XII of the Appendix. The equations for cation and anion doublet states are obtained from the triplet equations: Cation doublet states are obtained by replacing one of the unoccupied MO indices to infinitely separated orbital, e.g., $R_{i}^{\infty \dagger}$. Similarly, the electron-attached (anion doublet) states are written as an electron transfer from an infinitely separated orbital to one of the unoccupied orbitals like $\infty \rightarrow a$. Thus, the $R$ operators for the cation doublet and anion doublet are written as

$$
R^{\dagger}=\sum_{i} d_{i}^{\infty} R_{i}^{\infty \dagger \dagger}+\sum_{i j} \sum_{b} d_{i j}^{\infty b} R_{i j}^{\infty b^{\dagger}}
$$

and

$$
R^{\dagger}=\sum_{a} d_{\infty}^{a} R_{\infty}^{a \dagger}+\sum_{i} \sum_{a \neq b} d_{\infty j}^{a b} R_{\infty \infty j}^{a b \dagger},
$$

respectively. With the replacements to infinitely separated orbitals in Eqs. (34) and (35), the working equations for the cation doublet and anion doublet are obtained. Note that the matrix elements including indices $\infty$, such as $f_{i}^{\infty}, v_{i j}^{\infty a}$, etc., are zero.

\section{IMPLEMENTATION OF THE DIRECT SAC/SAC-CI ALGORITHM}

In the conventional nondirect program, the loops are driven with the labels, $I$, of excitation operators. The MO indices corresponding to the excitation operator are taken from the predefined labels of the excitation operators. This 
correspondence may be written as $S_{I}^{\dagger} \rightarrow S_{i j}^{a b \dagger}$ and is kept in the LABEL array, which is a $4 \times M$ matrix, where $M$ is the number of the selected operators and each operator has four $\mathrm{MO}$ indices.

Because the direct program drives loops with MO indices, we have to refer to the label of the excitation operator from the MO indices such as $S_{i j}^{a b \dagger} \rightarrow S_{I}^{\dagger}$. This correspondence is done by introducing INDEX arrays. The INDEX arrays are two-dimensional integer matrices, whose elements are excitation operator labels. We need six types of INDEX arrays: $\operatorname{INDEX}([i j],[a b]), \quad \operatorname{INDEX}([a b],[i j]), \quad \operatorname{INDEX}([i a],[j b])$, $\operatorname{INDEX}([j b],[i a]), \quad \operatorname{INDEX}([i b],[j a]), \quad$ and $\operatorname{INDEX}([j a],[i b])$, where the first elements are referred to as leading indices.

When we use the perturbation-selection technique, the length of the LABEL array is reduced and the INDEX arrays become sparse matrices. The INDEX arrays have no elements for the unselected excitation operators because the elements are appended only for the selected operators. Therefore, the INDEX arrays are compressed into a onedimensional vector together with the location array, Loc_LBL, that carries the information of the twodimensional structure of the original INDEX arrays. The loops are driven through the element of the INDEX vectors that assign the element of the LABEL array, which define the actual form of the excitation operator.

Let us explain the above algorithm using a simple example. We consider a system with two occupied ( $i$ and $j$ ) and two virtual ( $a$ and $b$ ) MOs. We assume that within ten possible excitation operators, the following six operators,

$$
\begin{aligned}
& S_{1}^{\dagger}=S_{i i}^{a a \dagger}, \quad S_{2}^{\dagger}=S_{i i}^{b b^{\dagger}}, \quad S_{3}^{\dagger}=S_{j j}^{a a^{\dagger}}, \\
& S_{4}^{\dagger}=S_{i j}^{b b^{\dagger}}, \quad S_{5}^{\dagger}=S_{i i}^{a b \dagger}, \quad S_{6}^{\dagger}=S_{i j}^{a b \dagger},
\end{aligned}
$$

were selected by the perturbation selection, discarding $S_{i j}^{a a^{\dagger}}$, $S_{i j}^{b a \dagger}, S_{j i}^{b b \dagger}$, and $S_{j j}^{a b \dagger}$. They actually have symmetry-adapted forms. ${ }^{1,2}$ The LABEL array is a $4 \times 6$ matrix

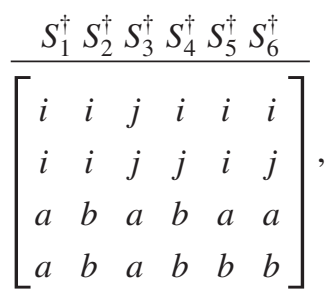

and the original $\operatorname{INDEX}([i j],[a b])$ array is a sparse matrix given by

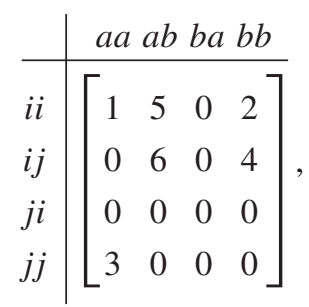

where only the selected elements are considered. This INDEX array is compressed into a one-dimensional INDEX vector

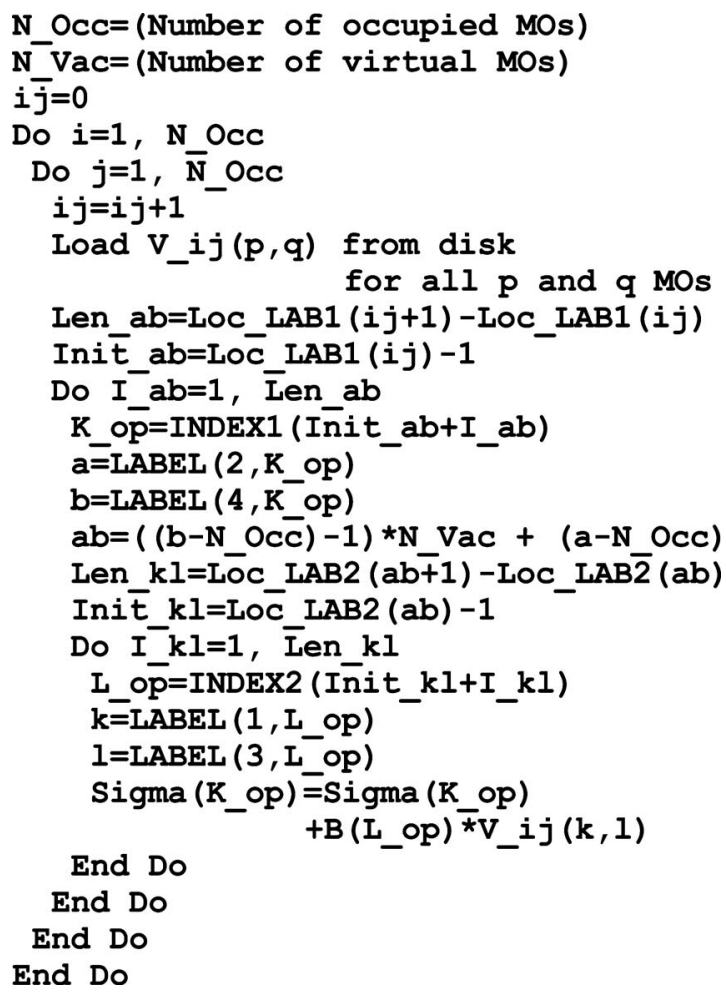

FIG. 1. Loop structure of direct SAC-CI for $\sigma_{i j}^{a b} \leftarrow[V]_{i j}^{k l} b_{k l}^{a b}$.

$\left[\begin{array}{llllll}1 & 5 & 2 & 6 & 4 & 3\end{array}\right]$,

with the information of the original array stored in the Loc_LBL array

$$
\left.\begin{array}{ccccc}
i i & i j & j i & j j & j j+1 \\
\hline[1 & 4 & 6 & 6 & 7
\end{array}\right] \text {, }
$$

where the element indicates the location in the INDEX vector that initiates the elements of the designated MO label, e.g., $i j$. The elements designated by the MO label $i j$ locate between fourth and fifth positions in INDEX. This can be schematized as

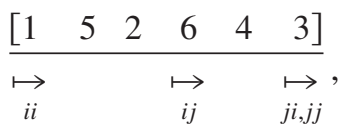

where an arrow, whose location is obtained from the Loc_LBL array, indicates the starting location of the designated MO label. The extra element of $j j+1$ is used for denoting the number of elements that belong to row $j j$. In the original INDEX array given by Eq. (38), the MO label $j i$ may be discarded from the beginning because of the symmetry of $S^{\dagger}$, e.g., $S_{j i}^{b a \dagger}=S_{i j}^{a b \dagger}$. The loop is driven through the INDEX vector given by Eq. (39) aided with the information stored in the Loc_LBL array.

We consider the system of the $N$-active orbitals and $M$-selected excitation operators. In the modified Davidson procedure, the sigma vector is constructed by the multiplication of the basis vector $\mathbf{b}$ and the intermediate arrays $[\boldsymbol{V}]$ as, for example, $\sigma_{i j}^{a b} \leftarrow[V]_{i j}^{k l} b_{k l}^{a b}$ (see the Appendix). The MO ERIs and intermediate arrays have been sorted in a desired 
TABLE I. Excitation energy, oscillator strength, and ionization potential of water.

\begin{tabular}{|c|c|c|c|c|c|c|}
\hline \multirow[b]{3}{*}{ State } & \multirow[b]{3}{*}{ Nature } & \multicolumn{2}{|c|}{ SAC-CI } & & & \multirow{3}{*}{$\begin{array}{c}\text { Expt. }^{b} \\
E_{\text {ex }}(\mathrm{eV})\end{array}$} \\
\hline & & \multicolumn{2}{|c|}{ Direct } & \multicolumn{2}{|c|}{ Nondirect } & \\
\hline & & $E_{\mathrm{ex}}(\mathrm{eV})$ & Osc $(\mathrm{au})^{\mathrm{a}}$ & $E_{\text {ex }}(\mathrm{eV})$ & Osc $(\mathrm{au})^{\mathrm{a}}$ & \\
\hline \multicolumn{7}{|c|}{ Singlet states } \\
\hline $1^{1} B_{1}$ & $\pi \rightarrow 3 s\left(\mathrm{OH}^{*}\right), \pi \rightarrow 4 s$ & 7.26 & 0.054 & 7.33 & 0.053 & $7.4,7.49$ \\
\hline $1{ }^{1} A_{2}$ & $\pi \rightarrow 3 p_{y}, \pi \rightarrow 4 p_{y}$ & 9.14 & $0^{\mathrm{c}}$ & 9.21 & $0^{\mathrm{c}}$ & 9.1 \\
\hline $2{ }^{1} A_{1}$ & $\pi \rightarrow 3 p_{x}, n \rightarrow 3 s$ & 9.58 & 0.060 & 9.67 & 0.053 & $9.67,9.73$ \\
\hline $2{ }^{1} B_{1}$ & $\pi \rightarrow 3 p_{z}$ & 9.72 & 0.008 & 9.78 & 0.008 & $10.01,9.996$ \\
\hline $3{ }^{1} A_{1}$ & $\pi \rightarrow 3 p_{x}, n \rightarrow 3 s$ & 9.89 & 0.038 & 9.96 & 0.044 & $10.17,10.14$ \\
\hline $3{ }^{1} B_{1}$ & $\pi \rightarrow 4 s, \pi \rightarrow 3 s$ & 11.12 & 0.007 & 11.20 & 0.007 & \\
\hline $1{ }^{1} B_{2}$ & $n \rightarrow 3 p_{y}, n \rightarrow 4 p_{y}$ & 11.50 & 0.014 & 11.59 & 0.014 & \\
\hline $2{ }^{1} A_{2}$ & $\pi \rightarrow 4 p_{y}, \pi \rightarrow 3 p_{y}$ & 11.57 & $0^{\mathrm{c}}$ & 11.68 & $0^{\mathrm{c}}$ & \\
\hline $4{ }^{1} B_{1}$ & $n \rightarrow 3 p_{x}$ & 11.97 & 0.000 & 12.04 & 0.000 & \\
\hline $4{ }^{1} A_{1}$ & $n \rightarrow 3 p_{z}$ & 12.01 & 0.004 & 12.09 & 0.004 & \\
\hline $5{ }^{1} A_{1}$ & $\pi \rightarrow 4 p_{x}$ & 12.80 & 0.004 & 12.87 & 0.004 & \\
\hline \multicolumn{7}{|c|}{ Triplet states } \\
\hline $1^{3} B_{1}$ & $\pi \rightarrow 3 s\left(\mathrm{OH}^{*}\right), \pi \rightarrow 4 s$ & 6.86 & $0^{\mathrm{c}}$ & 6.93 & $0^{\mathrm{c}}$ & $7.0,7.2$ \\
\hline $1^{3} A_{2}$ & $\pi \rightarrow 3 p_{y}, \pi \rightarrow 4 p_{y}$ & 8.98 & $0^{\mathrm{c}}$ & 9.08 & $0^{\mathrm{c}}$ & $8.9,9.1,9.2$ \\
\hline $1^{3} A_{1}$ & $n \rightarrow 3 s\left(\mathrm{OH}^{*}\right), n \rightarrow 4 s$ & 9.25 & $0^{\mathrm{c}}$ & 9.35 & $0^{\mathrm{c}}$ & 9.3 \\
\hline $2{ }^{3} A_{1}$ & $\pi \rightarrow 3 p_{x}, \pi \rightarrow 4 p_{x}$ & 9.47 & $0^{c}$ & 9.54 & $0^{\mathrm{c}}$ & $9.80,9.81$ \\
\hline $2{ }^{3} B_{1}$ & $\pi \rightarrow 3 p_{z}$ & 9.66 & $0^{\mathrm{c}}$ & 9.74 & $0^{\mathrm{c}}$ & 9.98 \\
\hline $3{ }^{3} B_{1}$ & $\pi \rightarrow 4 s, \pi \rightarrow 3 s$ & 10.86 & $0^{\mathrm{c}}$ & 10.94 & $0^{c}$ & \\
\hline $1{ }^{3} B_{2}$ & $n \rightarrow 3 p_{y}, n \rightarrow 4 p_{y}$ & 11.25 & $0^{\mathrm{c}}$ & 11.35 & $0^{\mathrm{c}}$ & \\
\hline $2^{3} A_{2}$ & $\pi \rightarrow 4 p_{y}, \pi \rightarrow 3 p_{y}$ & 11.33 & $0^{c}$ & 11.45 & $0^{\mathrm{c}}$ & \\
\hline $3{ }^{3} A_{1}$ & $n \rightarrow 3 p_{z}$ & 11.75 & $0^{\mathrm{c}}$ & 11.84 & $0^{\mathrm{c}}$ & \\
\hline $4{ }^{3} B_{1}$ & $n \rightarrow 3 p_{x}$ & 11.92 & $0^{c}$ & 12.00 & $0^{\mathrm{c}}$ & \\
\hline $4{ }^{3} A_{1}$ & $\pi \rightarrow 4 p_{x}$ & 12.20 & $0^{\mathrm{c}}$ & 12.26 & $0^{\mathrm{c}}$ & \\
\hline \multicolumn{7}{|c|}{ Cation states } \\
\hline $1^{2} B_{1}$ & $(\pi)^{-1}$ & 12.11 & 0.934 & 12.15 & 0.939 & 12.61 \\
\hline $1^{2} A_{1}$ & $(n)^{-1}$ & 14.41 & 0.937 & 14.48 & 0.939 & 14.73 \\
\hline $1^{2} B_{2}$ & $(\sigma)^{-1}$ & 18.78 & 0.949 & 18.84 & 0.952 & 18.55 \\
\hline
\end{tabular}

order on a disk. Consequently, at most, only the $N^{2}$ memory is required for the MO ERIs. A typical loop structure of the direct SAC-CI algorithm is shown in Fig. 1 for $\sigma_{i j}^{a b}$ $\leftarrow[V]_{i j}^{k l} a k l$. The intermediate array of $[V]_{i j}^{k l}$ for fixed $i$ and $j$ is loaded within the outer loops for $i$ and $j$, and these loops require $O\left(N^{2}\right)$ computations. The inner loops run only for nonzero elements of $\mathbf{b}$. The computations of the inner loops are $O(M)$. The total computational cost of the direct algorithm is $O\left(N^{2} \times M\right)$. In cases without perturbation selection, $M=O\left(N^{4}\right)$, so that this algorithm becomes $O\left(N^{6}\right)$, which agrees with the optimal cost of the singles and doubles coupled-cluster theories in the canonical MO basis.

\section{SAMPLE APPLICATIONS}

As test applications of the new direct SAC-CI program, we performed calculations for water $\left(\mathrm{H}_{2} \mathrm{O}\right)$, ethylene $\left(\mathrm{C}_{2} \mathrm{H}_{4}\right)$, pyrrole $\left(\mathrm{C}_{4} \mathrm{H}_{5} \mathrm{~N}\right)$, tetrathiomolybdate anion $\left(\mathrm{MoS}_{4}{ }^{2-}\right)$, and free-base porphin $\left(\mathrm{C}_{20} \mathrm{~N}_{4} \mathrm{H}_{14}\right)$. The molecular geometries were taken from the SAC-CI Guide. ${ }^{20}$ The basis sets were as follows: D95 $(d, p)$ with Rydberg $(2 s 2 p)$ (Ref. 29) on oxygen for $\mathrm{H}_{2} \mathrm{O}, \mathrm{D} 95(d, p)$ with Rydberg $(2 s 2 p 2 d)$ on carbon for
$\mathrm{C}_{2} \mathrm{H}_{4}$, D95(d) for $\mathrm{C}_{4} \mathrm{H}_{5} \mathrm{~N}$, LANL2DZ (Ref. 30) for $\mathrm{MoS}_{4}{ }^{2-}$, and D95 for free-base porphin. The perturbation selection with LevelThree threshold $\left(\lambda_{g}=1.0 \times 10^{-6}, \lambda_{e}=1.0 \times 10^{-7}\right)$ was used for all molecules since this is the recommended one. Additionally, LevelOne $\left(\lambda_{g}=1.0 \times 10^{-5}, \lambda_{e}=1.0 \times 10^{-6}\right)$ and LevelTwo $\left(\lambda_{g}=5.0 \times 10^{-6}, \lambda_{e}=5.0 \times 10^{-7}\right)$ calculations were carried out for free-base porphin. The levels of the selection can be selected with the GAUSSAIN03 keyword. The default setting was used for other conditions. We used an approximate variational method for solving the SAC-CI secular equation, in which the Hamiltonian matrix was symmetrized as $\tilde{H}_{I J}=\frac{1}{2}\left(H_{I J}+H_{J I}\right)$.

Note that in the conventional nondirect SAC-CI algorithm, an additional cutoff threshold was introduced for the unlinked terms to save computer time. The direct SAC-CI algorithm does not require such approximation, so that the direct and nondirect SAC-CI computations do not give completely identical results. Theoretically, the direct SAC-CI results should be more accurate than the conventional ones. Both versions of the SAC-CI programs run on the development version ${ }^{31}$ of GAUSSIAN03. All computations were car- 
TABLE II. Excitation energy, oscillator strength, and ionization potential of ethylene.

\begin{tabular}{|c|c|c|c|c|c|c|}
\hline \multirow[b]{3}{*}{ State } & \multicolumn{5}{|c|}{ SAC-CI } & \multirow{3}{*}{$\begin{array}{r}\text { Expt. } \\
E_{\mathrm{ex}}(\mathrm{eV})\end{array}$} \\
\hline & \multirow[b]{2}{*}{ Nature } & \multicolumn{2}{|c|}{ Direct } & \multicolumn{2}{|c|}{ Nondirect } & \\
\hline & & $E_{\mathrm{ex}}(\mathrm{eV})$ & Osc $(\mathrm{au})^{\mathrm{a}}$ & $E_{\text {ex }}(\mathrm{eV})$ & Osc $(\mathrm{au})^{\mathrm{a}}$ & \\
\hline \multicolumn{7}{|c|}{ Singlet states } \\
\hline $1{ }^{1} A_{g}$ & $\pi \rightarrow 3 p(\pi)$ & 8.18 & $0^{\mathrm{c}}$ & 8.33 & $0^{\mathrm{c}}$ & 8.28 \\
\hline $1{ }^{1} B_{1 g}^{8}$ & $\pi \rightarrow 3 p(\sigma)$ & 7.82 & $0^{\mathrm{c}}$ & 7.94 & $0^{\mathrm{c}}$ & 7.80 \\
\hline $2{ }^{1} B_{1 g}$ & $\sigma \rightarrow \pi^{*}$ & 8.74 & $0^{\mathrm{c}}$ & 8.94 & $0^{\mathrm{c}}$ & \\
\hline $1{ }^{1} B_{2 g}$ & $\pi \rightarrow 3 p(\sigma)$ & 7.82 & $0^{c}$ & 8.00 & $0^{\mathrm{c}}$ & 7.90 \\
\hline $1{ }^{1} B_{3 g}$ & $\sigma \rightarrow 3 s$ & 9.53 & $0^{\mathrm{c}}$ & 9.58 & $0^{\mathrm{c}}$ & 9.51 \\
\hline $1{ }^{1} A_{u}^{\circ}$ & $\pi \rightarrow 3 d(\pi)$ & 8.84 & $0^{\mathrm{c}}$ & 8.95 & $0^{\mathrm{c}}$ & \\
\hline $1{ }^{1} B_{1 u}$ & $\pi \rightarrow \pi^{*}$ & 8.12 & 0.349 & 8.21 & 0.366 & $\sim 8.0$ \\
\hline $2{ }^{1} B_{1 u}$ & $\pi \rightarrow 3 d(\pi)$ & 9.14 & 0.051 & 9.25 & 0.048 & 9.33 \\
\hline $1{ }^{1} B_{2 u}$ & $\pi \rightarrow 3 d(\delta)$ & 8.92 & 0.011 & 8.94 & 0.012 & 8.90 \\
\hline $1{ }^{1} B_{3 u}$ & $\pi \rightarrow 3 s$ & 7.15 & 0.092 & 7.33 & 0.093 & 7.11 \\
\hline $2{ }^{1} B_{3 u}$ & $\pi \rightarrow 3 d(\sigma)$ & 8.69 & 0.004 & 8.86 & 0.003 & 8.62 \\
\hline $3{ }^{1} B_{3 u}$ & $\pi \rightarrow 3 d(\delta)$ & 8.88 & 0.028 & 9.04 & 0.031 & 8.90 \\
\hline \multicolumn{7}{|c|}{ Triplet states } \\
\hline $1{ }^{3} A_{g}$ & $\pi \rightarrow 3 p(\pi)$ & 8.03 & $0^{\mathrm{c}}$ & 8.13 & $0^{\mathrm{c}}$ & 8.15 \\
\hline $1{ }^{3} B_{1 g}^{g}$ & $\pi \rightarrow 3 p(\sigma)$ & 7.79 & $0^{c}$ & 7.90 & $0^{\mathrm{c}}$ & 7.79 \\
\hline $2{ }^{3} B_{1 g}$ & $\sigma \rightarrow \pi^{*}$ & 8.42 & $0^{\mathrm{c}}$ & 8.60 & $0^{\mathrm{c}}$ & \\
\hline $1{ }^{3} B_{2 g}$ & $\pi \rightarrow 3 p(\sigma)$ & 7.76 & $0^{\mathrm{c}}$ & 7.94 & $0^{\mathrm{c}}$ & \\
\hline $1^{3} B_{3 g}$ & $\sigma \rightarrow 3 s$ & 9.53 & $0^{\mathrm{c}}$ & 9.58 & $0^{\mathrm{c}}$ & \\
\hline $1{ }^{3} A_{u}$ & $\pi \rightarrow 3 d(\pi)$ & 8.84 & $0^{\mathrm{c}}$ & 8.95 & $0^{\mathrm{c}}$ & \\
\hline $1{ }^{3} B_{1 u}$ & $\pi \rightarrow \pi^{*}$ & 4.40 & $0^{\mathrm{c}}$ & 4.50 & $0^{\mathrm{c}}$ & 4.36 \\
\hline $2{ }^{3} B_{1 u}$ & $\pi \rightarrow 3 d(\pi)$ & 8.95 & $0^{\mathrm{c}}$ & 9.07 & $0^{\mathrm{c}}$ & 8.86 \\
\hline $1{ }^{3} B_{2 u}$ & $\pi \rightarrow 3 d(\delta)$ & 8.92 & $0^{\mathrm{c}}$ & 8.94 & $0^{\mathrm{c}}$ & \\
\hline $1{ }^{3} B_{3 u}$ & $\pi \rightarrow 3 s$ & 7.02 & $0^{\mathrm{c}}$ & 7.12 & $0^{\mathrm{c}}$ & 6.98 \\
\hline $2{ }^{3} B_{3 u}$ & $\pi \rightarrow 3 d(\sigma)$ & 8.66 & $0^{\mathrm{c}}$ & 8.83 & $0^{\mathrm{c}}$ & 8.57 \\
\hline $3^{3} B_{3 u}$ & $\pi \rightarrow 3 d(\delta)$ & 8.84 & $0^{c}$ & 8.98 & $0^{\mathrm{c}}$ & \\
\hline \multicolumn{7}{|c|}{ Cation states } \\
\hline $1^{2} A_{g}$ & $\left(2 a_{g}\right)^{-1}$ & 14.56 & 0.917 & 14.68 & 0.924 & 14.66 \\
\hline $1^{2} B_{3 g}$ & $\left(1 b_{3 g}\right)^{-1}$ & 12.87 & 0.927 & 13.01 & 0.933 & 12.85 \\
\hline $1^{2} B_{1 u}$ & $\left(2 b_{1 u}\right)^{-1}$ & 19.33 & 0.848 & 19.48 & 0.852 & 19.23 \\
\hline $1^{2} B_{2 u}$ & $\left(1 b_{2 u}\right)^{-1}$ & 16.00 & 0.883 & 16.13 & 0.885 & 15.87 \\
\hline $1^{2} B_{3 u}$ & $\left(1 b_{3 u}\right)^{-1}$ & 10.34 & 0.949 & 10.36 & 0.952 & 10.51 \\
\hline
\end{tabular}

ried out with the Hewlett-Packard Integrity rx2620 server with the IA64 architecture.

\section{A. Small molecules}

For water and ethylene, the direct SAC-CI results are very close to the conventional nondirect ones, as seen from Tables I and II, and they reproduced well the experimental values. The average differences between the direct and nondirect results were $0.08 \mathrm{eV}$ for water and $0.12 \mathrm{eV}$ for ethylene. These differences are due to the cutoff of some small unlinked terms in the conventional program, which is acceptable for small molecules. Compared to the experiments, the direct results are worse than the nondirect ones, which we believe to be due to the basis set insufficiency.

Table III shows the singlet and triplet excitation energies, ionization energies, and electron affinities of pyrrole. These test calculations were done without including the Rydberg basis, so that we cannot expect to reproduce the ex- perimental values because the valence-Rydberg mixing was shown to be strong in some excited states of this molecule. ${ }^{22}$ Thus, we focus here only on the differences between the direct and nondirect SAC-CI results. The differences are particularly large for the electron affinities: The direct SAC-CI results are about $0.25-0.3 \mathrm{eV}$ lower than the nondirect ones, which arose from the accumulated effects of small unlinked terms cut off in the nondirect program. Since orbital reorganizations are expected to be important as well as electron correlations in the electron-attached states, the cutoff approximation in the unlinked term may be wrong.

Table IV shows the excitation energies of the tetrathiomolybdate anion. The orbital characters of this molecule are as follows: $1 t_{1}: \mathrm{S}(3 p)$ lone pair. $3 t_{2}: \operatorname{Mo}(5 p)$. $2 e: \operatorname{Mo}(d)$ $+\mathrm{S}(p)$ antibonding. $4 t_{2}: \operatorname{Mo}(d)+\mathrm{S}(p)$ antibonding.

The excitation energies of the direct SAC-CI method were lower than those of the nondirect method. The gap between the two methods depends on the nature of the exci- 
TABLE III. Excitation energy, ionization potential, and electron affinity of pyrrole.

\begin{tabular}{|c|c|c|c|c|c|c|}
\hline \multirow[b]{3}{*}{ State } & \multirow[b]{3}{*}{ Nature } & \multicolumn{2}{|c|}{ SAC-CI } & & & \multirow{3}{*}{$\begin{array}{c}\text { Expt. }^{\mathrm{b}} \\
E_{\mathrm{ex}}(\mathrm{eV})\end{array}$} \\
\hline & & \multicolumn{2}{|c|}{ Direct } & \multicolumn{2}{|c|}{ Nondirect } & \\
\hline & & $E_{\mathrm{ex}}(\mathrm{eV})$ & Osc. $(\mathrm{au})^{\mathrm{a}}$ & $E_{\mathrm{ex}}(\mathrm{eV})$ & Osc. $(\mathrm{au})^{\mathrm{a}}$ & \\
\hline \multicolumn{7}{|c|}{ Singlet states } \\
\hline $1^{1} A_{1}$ & $\pi_{2} \rightarrow \pi_{4}^{*}, \pi_{3} \rightarrow \pi_{5}^{*}$ & 6.68 & 0.004 & 6.53 & 0.003 & \\
\hline $1{ }^{1} A_{2}$ & $\pi_{3} \rightarrow \sigma^{*}$ & 7.62 & $0^{\mathrm{c}}$ & 7.83 & $0^{\mathrm{c}}$ & \\
\hline $1{ }^{1} B_{1}$ & $\pi_{2} \rightarrow \sigma^{*}$ & 8.40 & 0.011 & 8.65 & 0.011 & \\
\hline $1^{1} B_{2}$ & $\pi_{3} \rightarrow \pi_{4}^{*}$ & 6.90 & 0.190 & 7.00 & 0.214 & $6.2-6.5$ \\
\hline \multicolumn{7}{|c|}{ Triplet states } \\
\hline $1{ }^{3} A_{1}$ & $\pi_{2} \rightarrow \pi_{4}^{*}, \pi_{3} \rightarrow \pi_{5}^{*}$ & 5.61 & $0^{\mathrm{c}}$ & 5.61 & $0^{\mathrm{c}}$ & 5.10 \\
\hline $1{ }^{3} A_{2}$ & $\pi_{3} \rightarrow \sigma^{*}$ & 7.64 & $0^{c}$ & 7.87 & $0^{\mathrm{c}}$ & \\
\hline $1{ }^{3} B_{1}$ & $\pi_{2} \rightarrow \sigma^{*}$ & 8.12 & $0^{\mathrm{c}}$ & 8.42 & $0^{\mathrm{c}}$ & \\
\hline $1^{3} B_{2}$ & $\pi_{3} \rightarrow \pi_{4}^{*}$ & 4.47 & $0^{\mathrm{c}}$ & 4.52 & $0^{\mathrm{c}}$ & 4.20 \\
\hline \multicolumn{7}{|c|}{ Cation doublet states } \\
\hline $1^{2} A_{1}$ & $6 a_{1}(\sigma)^{-1}$ & 12.64 & 0.908 & 12.98 & 0.928 & $12.60,12.58$ \\
\hline $1^{2} A_{2}$ & $1 a_{2}\left(\pi_{3}\right)^{-1}$ & 7.85 & 0.934 & 7.93 & 0.953 & $8.02,8.21$ \\
\hline $1^{2} B_{1}$ & $2 b_{1}\left(\pi_{2}\right)^{-1}$ & 8.69 & 0.923 & 8.81 & 0.938 & $9.05,9.20$ \\
\hline $1^{2} B_{2}$ & $4 b_{2}(\sigma)^{-1}$ & 13.12 & 0.910 & 13.49 & 0.929 & 13.0 \\
\hline \multicolumn{7}{|c|}{ Anion doublet states } \\
\hline $1{ }^{2} A_{1}$ & $7 a_{1}\left(\sigma^{*}\right)^{+1}$ & 4.90 & & 5.14 & & \\
\hline $1^{2} A_{2}$ & $2 a_{2}\left(\pi_{5}^{*}\right)^{+1}$ & 4.93 & & 5.23 & & 3.45 \\
\hline $1^{2} B_{1}$ & $3 b_{1}\left(\pi_{4}^{*}\right)^{+1}$ & 3.76 & & 4.01 & & 2.36 \\
\hline $1^{2} B_{2}$ & $5 b_{2}\left(\sigma^{*}\right)^{+1}$ & 6.43 & & 6.68 & & \\
\hline
\end{tabular}

tation. The lower two states are valence-type excitations, from ligand lone pair to metal-ligand antibonding MO. For these states, the differences were around $0.2 \mathrm{eV}$. The next three states have a Rydberg nature, i.e., the excitations to molybdenum $5 p$ orbital. For these states, the differences were as large as $0.4 \mathrm{eV}$, showing that the neglected smaller contributions in the unlinked term could sum up to a large number. Such cases seem to occur when the coupling between the orbital reorganization and the electron correlation is large. The direct algorithm includes all such terms, and the results reproduced the overall experiments better than the nondirect one. When we reduce the cutoff threshold in the nondirect method, the results become close to those of the direct method, which will be shown in Sec. IV D.

\section{B. Free-base porphin}

The results for free-base porphin were summarized in Table V. The results for four threshold levels were given, but here we discuss only the LevelThree results because it is a recommended level. Except for the $1 B_{2 u}$ state, the excitation energies of the direct SAC-CI method were lower than those of the nondirect one. Consequently, the energy gap between the $1 B_{1 u}$ and $1 B_{2 u}$ states was increased, and the gap between the $1 B_{2 u}$ and $2 B_{1 u}$ states was reduced. The observed gap between $Q_{x}$ and $Q_{y}$ bands is $0.44 \mathrm{eV}$ and that between $Q_{y}$ and $B$ bands is $0.91 \mathrm{eV}$. The direct SAC-CI method improved the spectral shape by the reduction of the $1 B_{2 u}$ and $2 B_{1 u}$ gap.

TABLE IV. Excitation energy and oscillator strength of tetrathiomolybudate anion.

\begin{tabular}{|c|c|c|c|c|c|c|c|}
\hline \multirow[b]{3}{*}{ State } & \multicolumn{5}{|c|}{ SAC-CI } & \multicolumn{2}{|c|}{ Expt. $^{\mathrm{a}}$} \\
\hline & \multirow[b]{2}{*}{ Main configuration } & \multicolumn{2}{|c|}{ Direct } & \multicolumn{2}{|c|}{ Nondirect } & \multirow{2}{*}{$\begin{array}{r}E_{\mathrm{ex}} \\
(\mathrm{eV})\end{array}$} & \multirow[b]{2}{*}{ Osc. } \\
\hline & & $E_{\mathrm{ex}}(\mathrm{eV})$ & Osc. (au) & $E_{\mathrm{ex}}(\mathrm{eV})$ & Osc. (au) & & \\
\hline \multicolumn{8}{|c|}{ Singlet states } \\
\hline $1{ }^{1} T_{1}$ & $1 t_{1} \rightarrow 2 e$ & 2.11 & $0^{\mathrm{b}}$ & 2.30 & $0^{\mathrm{b}}$ & 2.37 & Weak \\
\hline $1{ }^{1} T_{2}$ & $1 t_{1} \rightarrow 2 e$ & 2.49 & 0.047 & 2.71 & 0.064 & 2.65 & 0.1 \\
\hline $1{ }^{1} E$ & $1 t_{1} \rightarrow 3 t_{2}$ & 3.17 & $0^{\mathrm{b}}$ & 3.61 & $0^{\mathrm{b}}$ & & \\
\hline $2{ }^{1} T_{1}$ & $1 t_{1} \rightarrow 3 t_{2}, 1 t_{1} \rightarrow 4 t_{2}$ & 3.19 & $0^{\mathrm{b}}$ & 3.60 & $0^{\mathrm{b}}$ & & \\
\hline $2{ }^{1} T_{2}$ & $1 t_{1} \rightarrow 3 t_{2}$ & 3.26 & 0.016 & 3.75 & 0.028 & 3.22 & Weak \\
\hline
\end{tabular}

${ }^{\mathrm{a}}$ Reference 39.

${ }^{\mathrm{b}}$ Symmetry allowed states are ${ }^{1} T_{2}$. 
TABLE V. Excitation energy and oscillator strength of free-base porphin.

\begin{tabular}{|c|c|c|c|c|c|c|c|}
\hline \multirow[b]{3}{*}{ State } & \multicolumn{5}{|c|}{ SAC-CI } & \multicolumn{2}{|c|}{ Expt. $^{\mathrm{a}}$} \\
\hline & \multirow[b]{2}{*}{ Nature } & \multicolumn{2}{|c|}{ Direct } & \multicolumn{2}{|c|}{ Nondirect } & \multirow{2}{*}{$\begin{array}{r}E_{\mathrm{ex}} \\
(\mathrm{eV})\end{array}$} & \multirow[b]{2}{*}{ Banc } \\
\hline & & $E_{\mathrm{ex}}(\mathrm{eV})$ & Osc. (au) & $E_{\mathrm{ex}}(\mathrm{eV})$ & Osc. (au) & & \\
\hline \multicolumn{8}{|c|}{ Level four } \\
\hline $1{ }^{1} B_{1 u}$ & $\pi \rightarrow \pi^{*}$ & 1.92 & 0.000 & 2.03 & 0.000 & 1.98 & $Q_{x}$ \\
\hline $1{ }^{1} B_{2 u}$ & $\pi \rightarrow \pi^{*}$ & 2.51 & 0.000 & 2.46 & 0.001 & 2.42 & $Q_{y}$ \\
\hline $2{ }^{1} B_{1 u}$ & $\pi \rightarrow \pi^{*}$ & 3.68 & 1.414 & 3.95 & 0.907 & 3.33 & $B$ \\
\hline $2{ }^{1} B_{2 u}$ & $\pi \rightarrow \pi^{*}$ & 3.79 & 1.820 & 4.19 & 1.726 & 3.65 & $N$ \\
\hline $3{ }^{1} B_{1 u}$ & $\pi \rightarrow \pi^{*}$ & 4.29 & 0.660 & 4.53 & 1.307 & & \\
\hline $3{ }^{1} B_{2 u}$ & $\pi \rightarrow \pi^{*}$ & 4.53 & 0.207 & 4.70 & 0.359 & $4.25-4.67$ & $L$ \\
\hline $4{ }^{1} B_{2 u}$ & $\pi \rightarrow \pi^{*}$ & 5.03 & 0.410 & 4.96 & 0.388 & $5.0-5.5$ & $M$ \\
\hline $4{ }^{1} B_{1 u}$ & $\pi \rightarrow \pi^{*}$ & 5.18 & 0.497 & 5.36 & 0.347 & & \\
\hline \multicolumn{8}{|c|}{ Level three } \\
\hline $1{ }^{1} B_{1 u}$ & $\pi \rightarrow \pi^{*}$ & 1.87 & 0.000 & 2.00 & 0.000 & 1.98 & $Q_{x}$ \\
\hline $1{ }^{1} B_{2 u}$ & $\pi \rightarrow \pi^{*}$ & 2.47 & 0.000 & 2.43 & 0.001 & 2.42 & $Q_{y}$ \\
\hline $2{ }^{1} B_{1 u}$ & $\pi \rightarrow \pi^{*}$ & 3.63 & 1.385 & 3.91 & 0.957 & 3.33 & $B$ \\
\hline $2{ }^{1} B_{2 u}$ & $\pi \rightarrow \pi^{*}$ & 3.74 & 1.799 & 4.13 & 1.745 & 3.65 & $N$ \\
\hline $3{ }^{1} B_{1 u}$ & $\pi \rightarrow \pi^{*}$ & 4.24 & 0.636 & 4.48 & 1.230 & & \\
\hline $3{ }^{1} B_{2 u}$ & $\pi \rightarrow \pi^{*}$ & 4.48 & 0.204 & 4.67 & 0.337 & $4.25-4.67$ & $L$ \\
\hline $4{ }^{1} B_{2 u}$ & $\pi \rightarrow \pi^{*}$ & 4.99 & 0.389 & 5.16 & 0.377 & $5.0-5.5$ & $M$ \\
\hline $4{ }^{1} B_{1 u}$ & $\pi \rightarrow \pi^{*}$ & 5.11 & 0.490 & 5.32 & 0.354 & & \\
\hline \multicolumn{8}{|c|}{ Level two } \\
\hline $1{ }^{1} B_{1 u}$ & $\pi \rightarrow \pi^{*}$ & 1.71 & 0.001 & 1.86 & 0.000 & 1.98 & $Q_{x}$ \\
\hline $1{ }^{1} B_{2 u}$ & $\pi \rightarrow \pi^{*}$ & 2.30 & 0.000 & 2.31 & 0.000 & 2.42 & $Q_{y}$ \\
\hline $2{ }^{1} B_{1 u}$ & $\pi \rightarrow \pi^{*}$ & 3.43 & 1.297 & 3.70 & 1.140 & 3.33 & $B$ \\
\hline $2{ }^{1} B_{2 u}$ & $\pi \rightarrow \pi^{*}$ & 3.54 & 1.646 & 3.86 & 1.722 & 3.65 & $N$ \\
\hline $3{ }^{1} B_{1 u}$ & $\pi \rightarrow \pi^{*}$ & 4.02 & 0.503 & 4.27 & 0.941 & & \\
\hline $3{ }^{1} B_{2 u}$ & $\pi \rightarrow \pi^{*}$ & 4.24 & 0.205 & 4.46 & 0.305 & $4.25-4.67$ & $L$ \\
\hline $4{ }^{1} B_{2 u}$ & $\pi \rightarrow \pi^{*}$ & 4.79 & 0.324 & 4.99 & 0.324 & $5.0-5.5$ & $M$ \\
\hline $4{ }^{1} B_{1 u}$ & $\pi \rightarrow \pi^{*}$ & 4.85 & 0.464 & 5.10 & 0.378 & & \\
\hline \multicolumn{8}{|c|}{ Level one } \\
\hline $1{ }^{1} B_{1 u}$ & $\pi \rightarrow \pi^{*}$ & 1.58 & 0.001 & 1.77 & 0.000 & 1.98 & $Q_{x}$ \\
\hline $1{ }^{1} B_{2 u}$ & $\pi \rightarrow \pi^{*}$ & 2.19 & 0.000 & 2.25 & 0.000 & 2.42 & $Q_{y}$ \\
\hline $2{ }^{1} B_{1 u}$ & $\pi \rightarrow \pi^{*}$ & 3.31 & 1.202 & 3.58 & 1.192 & 3.33 & $B$ \\
\hline $2{ }^{1} B_{2 u}$ & $\pi \rightarrow \pi^{*}$ & 3.40 & 1.526 & 3.71 & 1.690 & 3.65 & $N$ \\
\hline $3{ }^{1} B_{1 u}$ & $\pi \rightarrow \pi^{*}$ & 3.89 & 0.431 & 4.15 & 0.774 & & \\
\hline $3{ }^{1} B_{2 u}$ & $\pi \rightarrow \pi^{*}$ & 4.10 & 0.202 & 4.33 & 0.275 & $4.25-4.67$ & $L$ \\
\hline $4{ }^{1} B_{2 u}$ & $\pi \rightarrow \pi^{*}$ & 4.64 & 0.306 & 4.86 & 0.311 & $5.0-5.5$ & $M$ \\
\hline $4{ }^{1} B_{1 u}$ & $\pi \rightarrow \pi^{*}$ & 4.66 & 0.473 & 4.93 & 0.412 & & \\
\hline
\end{tabular}

${ }^{\mathrm{a}}$ Reference 40.

TABLE VI. Computational time (wall clock time) of SAC-CI calculations.

\begin{tabular}{lrr}
\hline \hline Molecule & Direct & Nondirect \\
\hline $\mathrm{H}_{2} \mathrm{O}$ & $52 \mathrm{~s}$ & $51 \mathrm{~s}$ \\
$\mathrm{C}_{2} \mathrm{H}_{4}$ & $9 \min 20 \mathrm{~s}$ & $11 \min 52 \mathrm{~s}$ \\
$\mathrm{MoS}_{4}{ }^{2-}$ & $12 \min 12 \mathrm{~s}$ & $18 \min 23 \mathrm{~s}$ \\
$\mathrm{C}_{4} \mathrm{H}_{5} \mathrm{~N}$ & $40 \min 15 \mathrm{~s}$ & $1 \mathrm{~h} 34 \min 17 \mathrm{~s}$ \\
Free-base porphin (level one) & $1 \mathrm{~h} 7 \min 20 \mathrm{~s}$ & $56 \min 11 \mathrm{~s}$ \\
Free-base porphin (level two) & $1 \mathrm{~h} 37 \min 54 \mathrm{~s}$ & $3 \mathrm{~h} 42 \min 28 \mathrm{~s}$ \\
Free-base porphin (level three) & $7 \mathrm{~h} 16 \min 35 \mathrm{~s}$ & $48 \mathrm{~h} 21 \min 07 \mathrm{~s}$ \\
Free-base porphin (level four) & $14 \mathrm{~h} 14 \min 50 \mathrm{~s}$ & $106 \mathrm{~h} 18 \min 59 \mathrm{~s}$ \\
\hline \hline
\end{tabular}

We also note the differences in the intensities between the two calculations. The most intense peak was the $2 B_{2 u}$ state. In the nondirect results the next intense state was $3 B_{1 u}$, whereas in the direct results the second intense state was $1 B_{1 u}$. In comparison with the observed spectrum, the intensity of the $3 B_{1 u}$ state with the nondirect method seems to be too strong, so that the direct algorithm improved not only the excitation energies, but also the absorption intensities. However, our assignments of the basic peaks are the same as those of the previous results. ${ }^{15,32}$ Since the basis set of the present calculations might be too poor, we postpone detailed arguments in a forthcoming paper.

We examined the convergence of the perturbationselection techniques. The differences between the LevelTwo and LevelThree results are about $0.15-0.25 \mathrm{eV}$. To confirm 
TABLE VII. Sets of cutoff thresholds for the unlinked terms of the nondirect method.

\begin{tabular}{|c|c|c|c|c|c|c|}
\hline & Keyword & Set 0 & Set 1 & Set 2 & Set 3 & Direct \\
\hline$\tau_{g}$ & CThreULS2G & $5.0 \times 10^{-3}$ & $5.0 \times 10^{-3}$ & $1.0 \times 10^{-3}$ & $1.0 \times 10^{-8}$ & 0 \\
\hline$\tau_{e}($ single $)$ & CThreULR1 & $5.0 \times 10^{-2}$ & $2.0 \times 10^{-2}$ & $1.0 \times 10^{-3}$ & $1.0 \times 10^{-8}$ & 0 \\
\hline$\tau_{e}$ (double) & CThreULR2 & $5.0 \times 10^{-2}$ & $2.0 \times 10^{-2}$ & $1.0 \times 10^{-3}$ & $1.0 \times 10^{-8}$ & 0 \\
\hline
\end{tabular}

the convergence of the perturbation-selection technique against the selection level, we performed additional calculations with tighter thresholds. (LevelFour: $\lambda_{g}=5 \times 10^{-7}$ and $\lambda_{e}=5 \times 10^{-8}$ ) The differences between the LevelThree and LevelFour results are about $0.05 \mathrm{eV}$, showing the converging behavior at higher levels of calculations toward the energies without selection.

\section{Computational time and space}

The computational times were summarized in Table VI. For $\mathrm{C}_{2} \mathrm{H}_{4}$ and $\mathrm{MoS}_{4}{ }^{2-}$, the direct method was slightly faster than the nondirect method. For $\mathrm{C}_{4} \mathrm{H}_{5} \mathrm{~N}$, the direct method was about 2.3 times faster than the nondirect method. Generally, the direct method was more efficient and the acceleration was more significant for larger dimensional computations. Actually, for free-base porphin, the acceleration factor was 6.7 for LevelThree calculations, 2.3 for LevelTwo calculations, and comparable to LevelOne calculations. Thus, the efficiency of the direct SAC-CI algorithm would extend the possibility of the SAC-CI method.

The number of nonzero MO ERIs of the free-base por-

TABLE VIII. Excitation energy and ionization potential of ethylene (in eV) with various levels of approximation.

\begin{tabular}{|c|c|c|c|c|c|c|c|}
\hline \multirow[b]{3}{*}{ State } & \multicolumn{6}{|c|}{ SAC-CI } & \multirow[b]{3}{*}{ Expt. } \\
\hline & \multirow[b]{2}{*}{ Nature } & \multicolumn{4}{|c|}{ Nondirect } & \multirow[b]{2}{*}{ Direct } & \\
\hline & & Set 0 & Set 1 & Set 2 & Set 3 & & \\
\hline \multicolumn{8}{|c|}{ Singlet states } \\
\hline $1{ }^{1} A_{g}$ & $\pi \rightarrow 3 p(\pi)$ & 8.33 & 8.18 & 8.18 & 8.18 & 8.18 & 8.28 \\
\hline $1{ }^{1} B_{1 g}^{8}$ & $\pi \rightarrow 3 p(\sigma)$ & 7.94 & 7.83 & 7.82 & 7.82 & 7.82 & 7.80 \\
\hline $2{ }^{1} B_{1 g}$ & $\sigma \rightarrow \pi^{*}$ & 8.94 & 8.80 & 8.74 & 8.74 & 8.74 & \\
\hline $1{ }^{1} B_{2 g}$ & $\pi \rightarrow 3 p(\sigma)$ & 8.00 & 7.84 & 7.82 & 7.82 & 7.82 & 7.90 \\
\hline $1{ }^{1} B_{3 g}{ }^{2 g}$ & $\sigma \rightarrow 3 s$ & 9.58 & 9.50 & 9.53 & 9.53 & 9.53 & 9.51 \\
\hline $1{ }^{1} A_{u}$ & $\pi \rightarrow 3 d(\pi)$ & 8.95 & 8.83 & 8.84 & 8.84 & 8.84 & \\
\hline $1{ }^{1} B_{1 u}$ & $\pi \rightarrow \pi^{*}$ & 8.21 & 8.13 & 8.12 & 8.12 & 8.12 & $\sim 8.0$ \\
\hline $2{ }^{1} B_{1 u}$ & $\pi \rightarrow 3 d(\pi)$ & 9.25 & 9.13 & 9.14 & 9.14 & 9.14 & 9.33 \\
\hline $1{ }^{1} B_{2 u}$ & $\pi \rightarrow 3 d(\delta)$ & 8.94 & 8.89 & 8.92 & 8.92 & 8.92 & 8.90 \\
\hline $1{ }^{1} B_{3 u}$ & $\pi \rightarrow 3 s$ & 7.33 & 7.18 & 7.15 & 7.15 & 7.15 & 7.11 \\
\hline $2{ }^{1} B_{3 u}$ & $\pi \rightarrow 3 d(\sigma)$ & 8.86 & 8.70 & 8.69 & 8.69 & 8.69 & 8.62 \\
\hline $3{ }^{1} B_{3 u}$ & $\pi \rightarrow 3 d(\delta)$ & 9.04 & 8.96 & 8.87 & 8.88 & 8.88 & 8.90 \\
\hline \multicolumn{8}{|c|}{ Triplet states } \\
\hline $1{ }^{3} A_{g}$ & $\pi \rightarrow 3 p(\pi)$ & 8.13 & 8.03 & 8.02 & 8.03 & 8.03 & 8.15 \\
\hline $1{ }^{3} B_{1 g}^{g}$ & $\pi \rightarrow 3 p(\sigma)$ & 7.90 & 7.80 & 7.79 & 7.79 & 7.79 & 7.79 \\
\hline $2{ }^{3} B_{1 g}$ & $\sigma \rightarrow \pi^{*}$ & 8.60 & 8.48 & 8.42 & 8.42 & 8.42 & \\
\hline $1{ }^{3} B_{2 g}$ & $\pi \rightarrow 3 p(\sigma)$ & 7.94 & 7.79 & 7.75 & 7.76 & 7.76 & \\
\hline $1{ }^{3} B_{3 g}$ & $\sigma \rightarrow 3 s$ & 9.58 & 9.50 & 9.53 & 9.53 & 9.53 & \\
\hline $1^{3} A_{u}^{g g}$ & $\pi \rightarrow 3 d(\pi)$ & 8.95 & 8.83 & 8.84 & 8.84 & 8.84 & \\
\hline $1^{3} B_{1 u}$ & $\pi \rightarrow \pi^{*}$ & 4.50 & 4.43 & 4.39 & 4.40 & 4.40 & 4.36 \\
\hline $2{ }^{3} B_{1 u}$ & $\pi \rightarrow 3 d(\pi)$ & 9.07 & 8.94 & 8.94 & 8.95 & 8.95 & 8.86 \\
\hline $1^{3} B_{2 u}$ & $\pi \rightarrow 3 d(\delta)$ & 8.94 & 8.89 & 8.92 & 8.92 & 8.92 & \\
\hline $1{ }^{3} B_{3 u}$ & $\pi \rightarrow 3 s$ & 7.12 & 7.05 & 7.02 & 7.02 & 7.02 & 6.98 \\
\hline $2{ }^{3} B_{3 u}$ & $\pi \rightarrow 3 d(\sigma)$ & 8.83 & 8.68 & 8.66 & 8.66 & 8.66 & 8.57 \\
\hline $3{ }^{3} B_{3 u}$ & $\pi \rightarrow 3 d(\delta)$ & 8.98 & 8.84 & 8.84 & 8.84 & 8.84 & \\
\hline \multicolumn{8}{|c|}{ Cation states } \\
\hline $1^{2} A_{g}$ & $\left(2 a_{g}\right)^{-1}$ & 14.68 & 14.53 & 14.55 & 14.56 & 14.56 & 14.66 \\
\hline $1^{2} B_{3 g}^{8}$ & $\left(1 b_{3 g}\right)^{-1}$ & 13.01 & 12.86 & 12.86 & 12.87 & 12.87 & 12.85 \\
\hline $1^{2} B_{1 u}$ & $\left(2 b_{1 u}\right)^{-1}$ & 19.48 & 19.34 & 19.33 & 19.33 & 19.33 & 19.23 \\
\hline $1^{2} B_{2 u}$ & $\left(1 b_{2 u}\right)^{-1}$ & 16.13 & 15.98 & 16.00 & 16.00 & 16.00 & 15.87 \\
\hline $1^{2} B_{3 u}$ & $\left(1 b_{3 u}\right)^{-1}$ & 10.36 & 10.29 & 10.34 & 10.34 & 10.34 & 10.51 \\
\hline Compu & tional time & $11 \min 52 \mathrm{~s}$ & $21 \min 05 \mathrm{~s}$ & $4 \mathrm{~h} 21 \min 33 \mathrm{~s}$ & $8 \mathrm{~h} 47 \min 31 \mathrm{~s}$ & $9 \min 20 \mathrm{~s}$ & \\
\hline
\end{tabular}


TABLE IX. Excitation energy of tetrathiomolybudate anion (in eV) with various levels of approximation.

\begin{tabular}{|c|c|c|c|c|c|c|c|}
\hline \multirow[b]{3}{*}{ State } & \multicolumn{6}{|c|}{ SAC-CI } & \multirow[b]{3}{*}{ Expt. } \\
\hline & \multirow[b]{2}{*}{ Main configuration } & \multicolumn{4}{|c|}{ Nondirect } & \multirow[b]{2}{*}{ Direct } & \\
\hline & & Set 0 & Set 1 & Set 2 & Set 3 & & \\
\hline \multicolumn{8}{|c|}{ Singlet state } \\
\hline $1^{1} T_{1}$ & $1 t_{1} \rightarrow 2 e$ & 2.30 & 2.09 & 2.09 & 2.11 & 2.11 & 2.37 \\
\hline $1^{1} T_{2}$ & $1 t_{1} \rightarrow 2 e$ & 2.71 & 2.51 & 2.47 & 2.49 & 2.49 & 2.65 \\
\hline $1^{1} E$ & $1 t_{1} \rightarrow 3 t_{2}$ & 3.61 & 3.41 & 3.17 & 3.17 & 3.17 & \\
\hline $2{ }^{1} T_{1}$ & $1 t_{1} \rightarrow 3 t_{2}, 1 t_{1} \rightarrow 4 t_{2}$ & 3.60 & 3.38 & 3.19 & 3.19 & 3.19 & \\
\hline $2{ }^{1} T_{2}$ & $1 t_{1} \rightarrow 3 t_{2}$ & 3.75 & 3.56 & 3.27 & 3.26 & 3.26 & 3.22 \\
\hline \multicolumn{2}{|c|}{ Computational time } & $18 \min 23 \mathrm{~s}$ & $38 \min 54 \mathrm{~s}$ & $21 \mathrm{~h} 30 \mathrm{~min} 07 \mathrm{~s}$ & $44 \mathrm{~h} 56 \min 25 \mathrm{~s}$ & $12 \min 12 \mathrm{~s}$ & \\
\hline
\end{tabular}

phin was 40755181 . Therefore, we need approximately 41 Mbyte memory for storing all the MO ERIs for the usage of the PROJR program in the nondirect algorithm. We have compressed the MO ERIs with the use of the point group symmetry of free-base porphin. Thus, the demand for memory was not severe for the present examples. On the other hand, the direct method does not require the storage of the MO ERIs on memory. This improvement would become significant when we apply the direct method to big biological molecules, which involve hundreds of orbitals without any symmetry.

\section{Unlinked terms}

As we have seen in some examples, the approximations in the unlinked terms used in the nondirect method have led to some degrees of errors. This error is due to the cutoff of smaller unlinked terms, and the amount is dependent on the cutoff threshold. In the present cases, the errors were approximately $0.1-0.3 \mathrm{eV}$. Considering the errors originating from other sources, the errors less than $0.3 \mathrm{eV}\left(\sim 0.01 E_{h}\right)$ might be practically acceptable for most cases. However, for $\mathrm{MoS}_{4}{ }^{2-}$, the error amounted to $0.4 \mathrm{eV}$, so that we have to show the method to reduce this error in the nondirect method.

If we reduce the cutoff thresholds in the unlinked terms, the nondirect results become close to those of the direct method. A severe problem is an increase in the computational time. So, optimal sets of thresholds are necessary to diminish the cutoff errors within permissible computational time. To find such optimal threshold, we investigated the effects of the cutoff of unlinked terms.

The cutoff scheme in the nondirect program is as follows. For the SAC ground state, we cutoff the unlinked terms generated by the product of the linked operators whose SDCI coefficients $C$ is smaller than a threshold $\tau_{g}$, and this $\tau_{g}$ value can be controlled with the CThreULS2G keyword categorized as "detailed keywords." 20 The unlinked terms of SAC-CI are generated by the product of the linked operators $R_{K}$ and $S_{I}$, where we include all the double excitation operators $S_{I}$ but we select important $R_{K}$ operators whose SDCI coefficients $d_{K}$ satisfy $d_{K}>\tau_{e}$, where the $\tau_{e}$ values for single and double excitation operators can be input by the keywords
CThreULR1 and CThreULR2, respectively. The direct SAC-CI program does not use this type of cutoff, so that the direct calculations correspond to $\tau_{g}=\tau_{e}=0$. To see the effect of this approximation in the nondirect method and to find an optimal set of thresholds, we examined four sets of thresholds for ethylene and tetrathiomolybudate anion. The thresholds are summarized in Table VII. Set 0 is the thresholds adopted as the default.

Table VIII shows the results for ethylene. The nondirect results for set 0 are the same as those given in Table II. We see that the results with set 2 thresholds reproduced the results of the direct method. However, this calculation takes too much computational time. In this case, set 1 thresholds seem to be a good compromise. It approximately reproduces the direct results and the computational time is twice of set 0 . If accurate results are necessary, this additional computational cost could be permissible.

Table IX shows the results for tetrathiomolybudate anion. The set 2 calculation also well reproduced the direct calculation even in this case. The computational time is, however, too large. It takes 70 times longer for the set 0 calculations. In this case, set 1 would be a realistic choice even though it still contains some degrees of errors. The discrepancy of $0.3 \mathrm{eV}$ in the $2 T_{2}$ state could be an acceptable range.

As a result of these test calculations, we recommend to use set 1 thresholds when one wants to get higher accuracy with the nondirect SAC-CI calculations of the released version. Of course, the direct SAC-CI version will be the best choice after the new version of the program is released. We note here that the non-direct method is still useful when one wants to use the general- $R \operatorname{method}^{7}$ and the high-spin codes. ${ }^{10}$

In Table IX, we further showed the results obtained with set 3 thresholds. These results are essentially the same as the direct ones. This means that the same calculations were performed with different algorithms. The efficiency of the direct algorithm is obvious. It is more than 200 times faster than the nondirect one for $\mathrm{MoS}_{4}{ }^{2-}$. This efficiency enables us to choose better thresholds for the perturbation selection of the linked operators. When we release the nondirect program, we selected LevelOne, LevelTwo, and LevelThree sets from the experiences in our previous researches and computational times. Since we now have more freedom due to the present 
TABLE X. Working equations for SAC. (We assume summation over all repeating indices.)

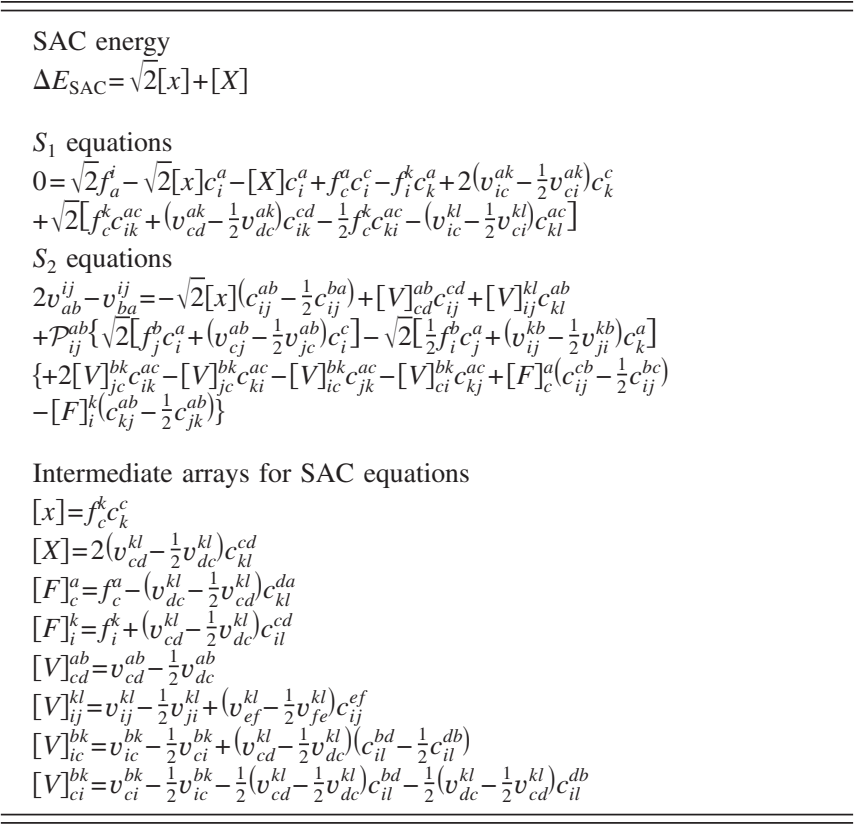

introduction of the direct method and the general advances in computer technology, we would be able to set up better sets of recommended thresholds for the perturbation selection of linked operators adopted in the SAC/SAC-CI program.

\section{CONCLUDING REMARKS}

We have developed a new computational algorithm, called direct algorithm, for the SAC/SAC-CI program. In the new direct SAC-CI SD- $R$ program, we could combine the MO-index direct algorithm with the perturbation-selection technique. The sparse nature of the matrices involved was fully utilized. The key of the present method was an efficient design of the index vectors and arrays that map the selected excitation operators. For the system of the $N$-active orbitals and $M$-selected excitation operators, the computational cost is $O\left(M \times N^{2}\right)$, which is optimal for singles and doubles theories with perturbation selection. In addition, an efficient usage of core memory has been achieved. Because of the achieved efficiency, the computer time became shorter, and the cutoff approximation in the unlinked terms with a given threshold, which was used in the conventional (nondirect) version of the program, became unnecessary.

The direct SAC-CI program has been applied to some test molecules. The direct SAC-CI algorithm was shown to be more efficient than the conventional one. Particularly, the computational speed was accelerated significantly for large dimensional computations with increasing accuracy of the results. The errors due to the cutoff approximation in the unlinked terms were minor in small molecules, but would become non-negligible for the systems where both of the correlations and orbital relaxations are important.

Thus, the direct SAC-CI program will provide theoretically more accurate results than before in a shorter computational time. This would extend the applicability of the SAC/SAC-CI methodology with increased accuracy. The
TABLE XI. Working equations for SAC-CI singlet excited states. (We assume summation over all repeating indices.)

$$
\begin{aligned}
& \text { Right-hand projection } \\
& \sigma_{0}=\sqrt{2} f_{c}^{k} b_{k}^{c}+2\left(v_{c d}^{k l}-\frac{1}{2} v_{d c}^{k l}\right) b_{k l}^{c d} \\
& \sigma_{i}^{a}=\sqrt{2} f_{i}^{a} b_{0}+[X] b_{i}^{a}-[F]_{i}^{k} b_{k}^{a}+[F]_{c}^{a} b_{i}^{c}+2\left(v_{i c}^{a k}-\frac{1}{2} v_{c i}^{a k}\right) b_{k}^{c} \\
& +2[G]_{c}^{k}\left(c_{k i}^{c a}-\frac{1}{2} c_{k i}^{a c}\right) \\
& +\sqrt{2}\left[f_{c}^{k}\left(b_{k i}^{c a}-\frac{1}{2} b_{i k}^{c a}\right)+\left(v_{k a}^{c d}-\frac{1}{2} v_{a k}^{c d}\right) b_{k i}^{c d}-\left(v_{c i}^{k l}-\frac{1}{2} v_{i c}^{k l}\right) b_{k l}^{c a}\right] \\
& \sigma_{i j}^{a b}=2\left(v_{i j}^{a b}-\frac{1}{2} v_{j i}^{a b}\right) b_{0}+[X]\left(b_{i j}^{a b}-\frac{1}{2} b_{i j}^{b a}\right)+[Y]\left(c_{i j}^{a b}-\frac{1}{2} c_{i j}^{b a}\right) \\
& +[V]_{i j}^{k l} b_{k l}^{a b}+[V]_{c d}^{a b} b_{i j}^{c d} \\
& +\mathcal{P}_{i j}^{a b}\left\{\sqrt{2}\left([F]_{i}^{a} b_{j}^{b}-\frac{1}{2}[F]_{i}^{b} b_{j}^{a}+[V]_{c j}^{b a} b_{i}^{c}-[V]_{j i}^{b k} b_{k}^{a}\right)+[G]_{c}^{a}\left(c_{i j}^{c b}\right.\right. \\
& \left.-\frac{1}{2} c_{i j}^{b c}\right)-[G]_{i}^{k}\left(c_{k j}^{a b}-\frac{1}{2} c_{j k}^{a b}\right) \\
& +2[V]_{c j}^{k b}\left(b_{k i}^{c a}-\frac{1}{2} b_{k i}^{a c}\right)-[V]_{c i}^{k b} b_{k j}^{c a}+[V]_{i c}^{k b} b_{k j}^{a c}+[F]_{c}^{a}\left(b_{i j}^{c b}-\frac{1}{2} b_{i j}^{b c}\right) \\
& \left.-[F]_{i}^{k}\left(b_{k j}^{a b}-\frac{1}{2} b_{j k}^{a b}\right)\right\} \\
& \tau_{0}=b_{0} \\
& \tau_{i}^{a}=b_{i}^{a} \\
& \tau_{i j}^{a b}=b_{i j}^{a b}-\frac{1}{2} b_{i j}^{b a} \\
& \sigma^{0}=\sqrt{2} b_{c}^{k} f_{k}^{c}+2 b_{c d}^{k l}\left(v_{k l}^{c d}-\frac{1}{2} v_{l k}^{c d}\right) \\
& \sigma_{a}^{i}=\left(\sqrt{2} b^{0}+[W]\right) f_{a}^{i}+b_{a}^{i}[X]-b_{a}^{k}[F]_{k}^{i}+b_{c}^{i}[F]_{c}^{a}+2 b_{c}^{k}\left(v_{k a}^{c i}-\frac{1}{2} v_{a k}^{c i}\right) \\
& +2\left(v_{a c}^{i k}-\frac{1}{2} v_{c a}^{i k}\right)[I]_{k}^{c} \\
& +\sqrt{2}\left\{\left(b_{c a}^{k i}-\frac{1}{2} b_{c a}^{i k}\right)[F]_{k}^{c}+b_{c d}^{i k}[V]_{a k}^{c d}-b_{c a}^{k l}[V]_{k l}^{c i}-\frac{1}{2} f_{a}^{k}[I]_{k}^{i}-\left(v_{a l}^{i k}\right.\right. \\
& \left.\left.-\frac{1}{2} v_{l a}^{i k}\right)[I]_{k}^{l}-\frac{1}{2} f_{c}^{i}[I]_{a}^{c}+\left(v_{a c}^{i d}-\frac{1}{2} v_{c a}^{i d}\right)[I]_{d}^{c}\right\} \\
& \sigma_{a b}^{i j}=2 b^{0}\left(v_{a b}^{i j}-\frac{1}{2} v_{b a}^{i j}\right)+\left(b_{a b}^{i j}-\frac{1}{2} b_{b a}^{i j}\right)[X]+[W]\left(v_{a b}^{j i}-\frac{1}{2} v_{b a}^{j i}\right) \\
& +b_{a b}^{k l}[V]_{k l}^{i j}+b_{c d}^{i j}[V]_{a b}^{c d} \\
& +\mathcal{P}_{i j}^{a b}\left\{\sqrt{2}\left[b_{a}^{i} f_{b}^{j}-\frac{1}{2} b_{a}^{j} f_{b}^{i}+b_{c}^{i}\left(v_{a b}^{c j}-\frac{1}{2} v_{a b}^{j c}\right)-b_{a}^{k}\left(v_{k b}^{i j}-\frac{1}{2} v_{b k}^{i j}\right)\right]\right. \\
& +2\left(b_{a c}^{i k}-\frac{1}{2} b_{a c}^{k i}\right)[V]_{b k}^{j c}-b_{a c}^{j k}[V]_{b k}^{i c} \\
& +b_{c a}^{j k}[V]_{k b}^{i c}+\left(b_{c b}^{i j}-\frac{1}{2} b_{b c}^{i j}\right)[F]_{a}^{c}-\left(b_{a b}^{k j}-\frac{1}{2} b_{a b}^{j k}\right)[F]_{k}^{i}-\left(v_{b c}^{j i}-\frac{1}{2} v_{c b}^{j i}\right) \\
& \left.\times[I]_{a}^{c}-\left(v_{j k}^{b a}-\frac{1}{2} v_{k j}^{b a}\right)[I]_{k}^{i}\right\} \\
& \tau^{0}=b^{0} \\
& \tau_{a}^{i}=b_{a}^{i} \\
& \tau_{a b}^{i j}=b_{a b}^{i j}-\frac{1}{2} b_{b a}^{i j}
\end{aligned}
$$

Intermediate arrays for SAC-CI singlet states

$[X]=2\left(v_{c d}^{k l}-\frac{1}{2} v_{d c}^{k l}\right) c_{k l}^{c d}$

$[Y]=\sqrt{2} f_{c}^{k} b_{k}^{c}+2\left(v_{c d}^{k l}-\frac{1}{2} v_{d c}^{k l}\right) b_{k l}^{c d}$

$[W]=\left(b_{c d}^{k l}-\frac{1}{2} b_{c d}^{l k}\right) c_{k l}^{c d}$

$[F]_{j}^{i}=f_{j}^{i}-\left(v_{c d}^{i k}-\frac{1}{2} v_{c d}^{k i}\right) c_{j k}^{c d}$

$[F]_{b}^{a}=f_{b}^{a}-\left(v_{c b}^{k l}-\frac{1}{2} v_{b c}^{k l}\right) c_{k l}^{c a}$

$[F]_{i}^{a}=f_{i}^{a}+f_{c}^{k}\left(c_{i k}^{a c}-\frac{1}{2} c_{i k}^{c a}\right)-\left(v_{i c}^{k l}-\frac{1}{2} v_{c i}^{k l}\right) c_{k l}^{a c}+\left(v_{c d}^{a k}-\frac{1}{2} v_{c d}^{k a}\right) c_{i k}^{c d}$

$[G]_{j}^{i}=\frac{\sqrt{2}}{2} f_{c}^{i} b_{j}^{c}+\sqrt{2}\left(v_{c j}^{k i}-\frac{1}{2} v_{j c}^{k i}\right) b_{k}^{c}+\left(v_{c d}^{k i}-\frac{1}{2} v_{c d}^{i k}\right) b_{k j}^{c d}$

$[G]_{b}^{a}=-\frac{\sqrt{2}}{2} f_{b}^{k} b_{k}^{a}+\sqrt{2}\left(v_{b c}^{a k}-\frac{1}{2} v_{c b}^{a k}\right) b_{k}^{c}-\left(v_{c b}^{k l}-\frac{1}{2} v_{b c}^{k l}\right) b_{k l}^{c a}$

$[G]_{a}^{i}=\left(v_{a c}^{i k}-\frac{1}{2} v_{c a}^{i k}\right) b_{k}^{c}$

$[I]_{j}^{i}=b_{c d}^{k i}\left(c_{k j}^{c d}-\frac{1}{2} c_{j k}^{c d}\right)+b_{c d}^{i k}\left(c_{j k}^{c d}-\frac{1}{2} c_{k j}^{c d}\right)$

$[I]_{b}^{a}=b_{c b}^{k l}\left(c_{k l}^{c a}-\frac{1}{2} c_{k l}^{a c}\right)+b_{b c}^{k l}\left(c_{k l}^{a c}-\frac{1}{2} c_{k l}^{c a}\right)$

$[I]_{i}^{a}=b_{k}^{c}\left(c_{k i}^{c a}-\frac{1}{2} c_{k i}^{a c}\right)$

$[V]_{c i}^{a b}=v_{c i}^{a b}-\frac{1}{2} v_{i c}^{a b}+\left(v_{c d}^{a k}-\frac{1}{2} v_{d c}^{a k}\right)\left(c_{i k}^{b d}-\frac{1}{2} c_{i k}^{d b}\right)-\frac{1}{2}\left(v_{d c}^{k b}-\frac{1}{2} v_{c d}^{k b}\right) c_{i k}^{a d}$

$-\frac{1}{2}\left(v_{c d}^{k b}-\frac{1}{2} v_{d c}^{k b}\right) c_{i k}^{d a}+\frac{1}{2}\left(v_{c i}^{k l}-\frac{1}{2} v_{i c}^{k l}\right)_{k l}^{a b}$

$[V]_{i j}^{a k}=v_{i j}^{a k}-\frac{1}{2} v_{i j}^{k a}+\left(v_{j c}^{k l}-\frac{1}{2} v_{c j}^{k l}\right)\left(c_{i l}^{a c}-\frac{1}{2} c_{i l}^{c a}\right)-\frac{1}{2}\left(v_{i c}^{k l}-\frac{1}{2} v_{c i}^{k l}\right) c_{j l}^{a c}$

$-\frac{1}{2}\left(v_{c i}^{k l}-\frac{1}{2} v_{i c}^{k l}\right) c_{j l}^{c a}+\frac{1}{2}\left(v_{c d}^{a k}-\frac{1}{2} v_{d c}^{a k}\right) c_{i j}^{c d}$

$[V]_{i j}^{k l}=\left(v_{i j}^{k l}-\frac{1}{2} v_{j i}^{k l}\right)+\frac{1}{2}\left(v_{c d}^{k l}-\frac{1}{2} v_{d c}^{k l}\right) c_{i j}^{c d}$

$[V]_{c d}^{a b}=\left(v_{c d}^{a b}-\frac{1}{2} v_{c d}^{b a}\right)+\frac{1}{2}\left(v_{c d}^{k l}-\frac{1}{2} v_{c d}^{l k}\right) c_{k l}^{a b}$

$[V]_{c i}^{k a}=\left(v_{c i}^{k a}-\frac{1}{2} v_{c i}^{a k}\right)+\left(v_{c d}^{k l}-\frac{1}{2} v_{d c}^{k l}\right)\left(c_{i l}^{a d}-\frac{1}{2} c_{i l}^{d a}\right)$

$[V]_{i c}^{k a}=-\left(v_{i c}^{k a}-\frac{1}{2} v_{c i}^{k a}+\frac{1}{2}\left(v_{c d}^{k l}-\frac{1}{2} v_{d c}^{k l}\right) c_{i l}^{a d}+\frac{1}{2}\left(v_{d c}^{k l}-\frac{1}{2} v_{c d}^{k l}\right) c_{i l}^{d a}\right.$

merits of the direct SAC-CI program will be recognized much more clearly for studies of excited-state geometries and potential energy surfaces. The SAC-CI energy gradient method based on the direct algorithm is certainly necessary for these studies. The idea of the direct SAC-CI method and the usage of sparse matrix techniques can be applied to the SAC-CI energy gradient method, which is in progress in our research institute. 
TABLE XII. Working equations for SAC-CI triplet states. [We assume summation over all repeating indices; however, $b$ with identical virtual orbitals must be dropped (e.g., $\left.v_{c d}^{k i} b_{j k}^{c d} \equiv \Sigma_{k} \Sigma_{c(\neq) d} v_{c d}^{k i} c_{j k}^{c d}\right)$.]

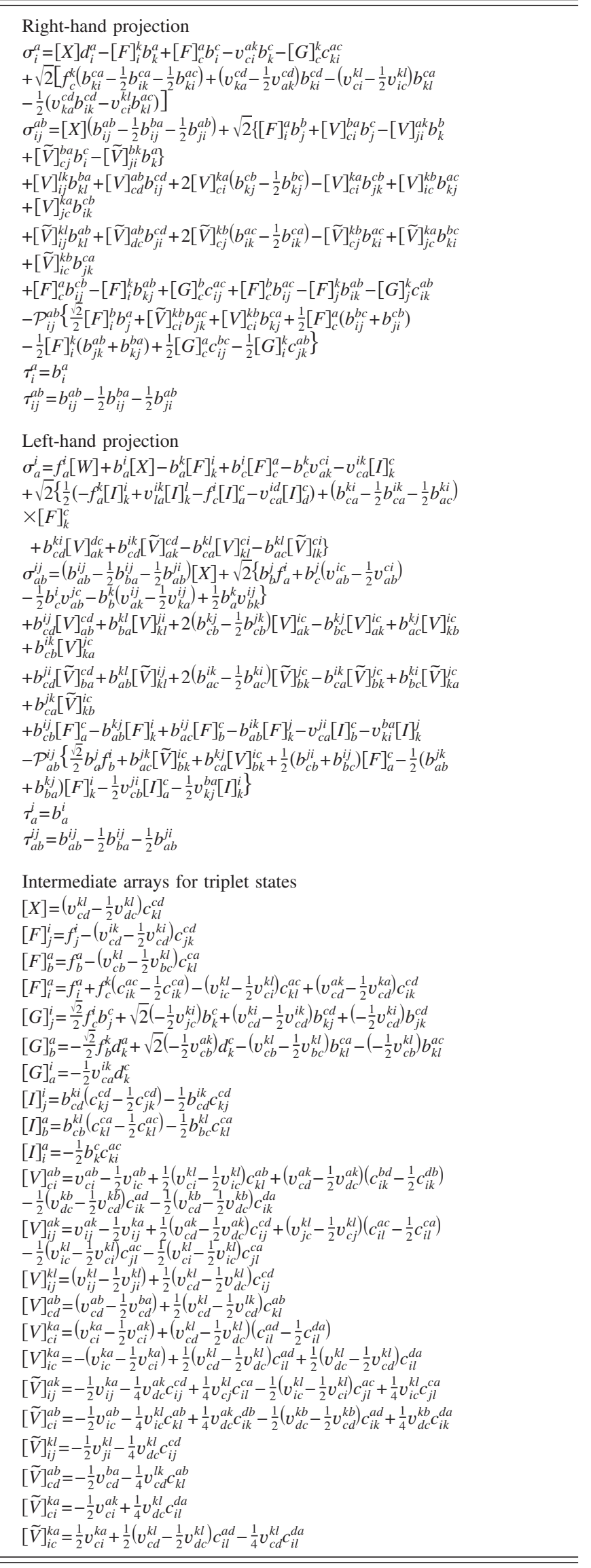

\section{ACKNOWLEDGMENTS}

The discussions in the SAC-CI meeting of our laboratory were very valuable. We thank the members of the SAC-CI meeting, particularly Dr. M. Hada and Dr. M. Ehara. This work has been supported by the grant for creative scientific research from the Ministry of Education, Science, Culture, and Sports of Japan.

\section{APPENDIX: WORKING EQUATIONS}

We summarize here the working equations for the singlet SAC equation in Table $\mathrm{X}$ and for the singlet and triplet SAC-CI equations in Tables XI and XII, respectively. The working equations for the SAC-CI ionized and electron attached doublet states are obtained by replacing the orbitals by infinitely separated orbitals as in Eqs. (34) and (35), respectively. Note that the matrix elements including indices $\infty$, such as $f_{i}^{\infty}, v_{i j}^{\infty a}$, etc., are zero.

${ }^{1}$ H. Nakatusji and K. Hirao, J. Chem. Phys. 68, 2053 (1978).

${ }^{2}$ H. Nakatsuji, Chem. Phys. Lett. 59, 362 (1978); 67, 329 (1979); 67, 334 (1979).

${ }^{3}$ H. Nakatsuji, Acta Chim. Hung. 129, 719 (1992); Computational Chemistry: Reviews of Current Trends, edited by J. Leszczynski (World Scientific, Singapore, 1997), Vol. 2, pp. 62-124; M. Ehara, M. Ishida, K. Toyota, and H. Nakatsuji, in Reviews of Modern Quantum Chemistry: A Celebration of the Contributions of Robert G. Parr, edited by K. D. Sen (World Scientific, Singapore, 2002), pp. 293-319; M. Ehara, J. Hasegawa, and H. Nakatsuji, in Theory and Application of Computational Chemistry, The First 40 Years, edited by C. E. Dykstra, G. Frenking, K. S. Kim, and G. E. Scuseria (Elsevier, Oxford, 2005), Chap. 39, pp. 10991141.

${ }^{4}$ D. Mukherjee and P. K. Mukherjee, Chem. Phys. 39, 325 (1979); H. Koch and P. Jorgensen, J. Chem. Phys. 93, 3333 (1990); H. Koch, H. J. Aa. Jansen, T. Helgaker, and P. Jorgensen, ibid. 93, 3345 (1990).

${ }^{5}$ J. Geersten, M. Rittby, and R. J. Bartlett, Chem. Phys. Lett. 164, 57 (1989); J. F. Stanton and R. J. Bartlett, J. Chem. Phys. 98, 7029 (1993).

${ }^{6}$ H. Nakatsuji, Program System for SAC and SAC-CI Calculations, Program Library No. 146(Y4/SAC), Data Processing Center of Kyoto University, 1985; Program Library SAC85 (No. 1396), Computer Center of the Institute for Molecular Science, Okazaki, Japan, 1986.

${ }^{7}$ H. Nakatsuji, Chem. Phys. Lett. 177, 331 (1991).

${ }^{8}$ H. Nakatsuji, J. Chem. Phys. 83, 713 (1985).

${ }^{9}$ H. Nakatsuji, J. Chem. Phys. 83, 5743 (1985); Theor. Chim. Acta 71, 201 (1987); J. Chem. Phys. 94, 6716 (1991); 95, 4296 (1991).

${ }^{10}$ H. Nakatsuji and M. Ehara, J. Chem. Phys. 98, 7179 (1993); 99, 1952 (1993).

${ }^{11}$ T. Nakajima and H. Nakatsuji, Chem. Phys. Lett. 280, 79 (1997); Chem. Phys. 242, 177 (1998).

${ }^{12}$ H. Nakatsuji, T. Miyahara, and R. Fukuda, J. Chem. Phys. 126, 084104 (2007).

${ }^{13}$ M. J. Frisch, G. W. Trucks, H. B. Schlegel et al., GAUSSIAN03, Gaussian, Inc., Pittsburgh, PA, 2003.

${ }^{14}$ H. Nakatsuji, Chem. Phys. 75, 425 (1983).

${ }^{15}$ H. Nakatsuji, J. Hasegawa, and M. Hada, J. Chem. Phys. 104, 2321 (1996).

${ }^{16}$ K. Hirao, J. Chem. Phys. 79, 5000 (1983).

${ }^{17}$ B. Roos, Chem. Phys. Lett. 15, 153 (1972).

${ }^{18}$ J. A. Pople, R. Krishnan, H. B. Schlegel, and J. S. Binkley, Int. J. Quantum Chem. 14, 545 (1978); R. J. Bartlett and G. D. Purvis, ibid. 14, 561 (1978).

${ }^{19}$ G. E. Scuseria, C. L. Janssen, and H. F. Schaefer III, J. Chem. Phys. 89, 7382 (1988).

${ }^{20}$ H. Nakatsuji, M. Hada, M. Ehara et al., SAC-CI Guide, 2005. (a pdf file is available at http://www.qcri.or.jp/sacci/).

${ }^{21}$ K. Ueda, M. Hoshino, T. Tanaka et al., Phys. Rev. Lett. 94, 243004 (2005); M. Ehara, H. Nakatsuji, M. Matsumoto et al., J. Chem. Phys. 124, 124311 (2006); M. Ehara, K. Kuramoto, H. Nakatsuji, M. Hoshino, T. Tanaka, M. Kitajima, H. Tanaka, Y. Tamenori, and K. Ueda, ibid. 125, 114303 (2006); R. Sankari, M. Ehara, H. Nakatsuji, A. De Fanis, H. 
Aksela, S. L. Sorensen, M. N. Piancastelli, E. Kukk, and K. Ueda, Chem. Phys. Lett. 422, 51 (2006); T. Tanaka, R. Feifel, H. Tanaka et al., ibid. 435, 182 (2007).

${ }^{22}$ J. Wan, J. Meller, M. Hada, M. Ehara, and H. Nakatsuji, J. Chem. Phys. 113, 7853 (2000); J. Wan, M. Ehara, M. Hada, and H. Nakatsuji, ibid. 113, 5245 (2000); J. Wan, M. Hada, M. Ehara, and H. Nakatsuji, ibid. 114, 5117 (2001); 114, 842 (2001).

${ }^{23}$ M. Ehara, M. Ishida, and H. Nakatsuji, J. Chem. Phys. 114, 8990 (2001); 117, 3248 (2002); M. Ishida, K. Toyota, M. Ehara, H. Nakatsuji, and M. J. Frisch, ibid. 120, 2593 (2004); M. Ehara, Y. Ohtsuka, H. Nakatsuji, M. Takahashi, and Y. Udagawa, ibid. 122, 234319 (2005); S. Arulmozhiraja, R. Fukuda, M. Ehara, and H. Nakatsuji, ibid. 124, 034312 (2006); T. Nakajima, S. Hane, and K. Hirao, ibid. 124, 224307 (2006); B. Saha, M. Ehara, and H. Nakatsuji, ibid. 125, 014316 (2006).

${ }^{24}$ H. Nakatsuji, J. Hasegawa, and K. Ohkawa, Chem. Phys. Lett. 296, 499 (1998); J. Hasegawa, K. Ohkawa, and H. Nakatsuji, J. Phys. Chem. B 102, 10410 (1998); J. Hasegawa and H. Nakatsuji, ibid. 102, 10420 (1998); T. Miyahara, Y. Tokita, and H. Nakatsuji, ibid. 105, 7341 (2001); A. K. Das, J. Hasegawa, T. Miyahara, M. Ehara, and H. Nakatsuji, J. Comput. Chem. 24, 1421 (2003).

${ }^{25}$ J. Hasegawa, M. Isshiki, K. Fujimoto, and H. Nakatsuji, Chem. Phys. Lett. 410, 90 (2005); K. Fujimoto, J. Hasegawa, S. Hayashi, S. Kato, and H. Nakatsuji, ibid. 414, 239 (2005); K. Fujimoto, J. Hasegawa, S. Hayashi, and H. Nakatsuji, ibid. 432, 252 (2006); J. Hasegawa and H. Nakatsuji, Chem. Lett. 34, 1242 (2005); N. Nakatani, J. Hasegawa, and H. Nakatsuji, J. Am. Chem. Soc. 129, 8756 (2007).

${ }^{26}$ C. M. Reeves, Commun. ACM 9, 276 (1966); I. L. Cooper and R. McWeeny, J. Chem. Phys. 45, 226 (1966); B. T. Sutcliffe, ibid. 45, 235 (1966).
${ }^{27}$ B. R. Gilson, PROJR, Program 218, Quantum Chemistry Program Exchange, Indiana University 1972.

${ }^{28}$ K. Hirao and H. Nakatsuji, J. Comput. Phys. 45, 246 (1982).

${ }^{29}$ T. H. Dunning, Jr. and P. J. Hay, in Modern Theoretical Chemistry, edited by H. F. Schaefer III (Plenum, New York, 1977), Vol. 3, p. 1.

${ }^{30}$ P. J. Hay and W. R. Wadt, J. Chem. Phys. 82, 270 (1985); 82, 284 (1985); 82, 299 (1985).

${ }^{31}$ M. J. Frisch, G. W. Trucks, H. B. Schlegel et al., GAUsSIAN Development Version, Revision E.05, Gaussian, Inc., Wallingford, CT, 2006.

${ }^{32}$ Y. Tokita, J. Hasegawa, and H. Nakatsuji, J. Phys. Chem. A 102, 1843 (1998).

${ }^{33}$ N. W. Winter, W. A. Goddard III, and F. W. Bobrowicz, J. Chem. Phys. 62, 4325 (1975).

${ }^{34}$ W. Mayer, Int. J. Quantum Chem. 5, 341 (1971).

${ }^{35}$ R. S. Mulliken, J. Chem. Phys. 66, 2448 (1977); D. G. Wilden and J. Comer, J. Phys. B 13, 1009 (1980); R. McDiarmid, J. Phys. Chem. 84, 64 (1980); A. Gedanken, N. A. Kuebler, and M. A. Robin, J. Chem. Phys. 76, 46 (1982); B. A. Williams and T. A. Cool, ibid. 94, 6358 (1991); W. H. Flicker, O. A. Mosher, and A. Kuppermann, Chem. Phys. Lett. 36, 56 (1975); M. H. Palmer, A. J. Beveridge, I. C. Walker, and T. Abuain, Chem. Phys. 102, 63 (1986).

${ }^{36}$ D. W. Turner, C. Baker, A. D. Baker, and C. R. Brundle, Molecular Photoelectron Spectroscopy (Wiley-Interscience, London, 1970).

${ }^{37}$ M. H. Palmer, I. C. Walker, and M. F. Guest, Chem. Phys. 238, 179 (1998).

${ }^{38}$ A. Modelli and P. D. Burrow, J. Phys. Chem. A 108, 5271 (2004).

${ }^{39}$ A. Müller and E. Diemann, Chem. Phys. Lett. 9, 369 (1971).

${ }^{40}$ L. Edwards and D. H. Dolphin, J. Mol. Spectrosc. 38, 16 (1971). 